\title{
Evidence of the Strong Nexus between Economic, Social, Business, and Political Indicators across the World
}

\section{Elias Sanidas*}

Department of Economics, Seoul National University, South Korea

\begin{abstract}
There are at least four levels of socio-economic and political development: institutional environment, micro business governance, economic resource allocation and employment, and finally culture or informal long term institutions. In our study we use methods of multivariate analysis such as multi-dimensional scaling and hierarchical clustering to provide evidence that these four levels (at least) of embeddedness exist as predicted; and that according to this model, countries are clustered together in such a way that we can reasonably accept an overall gauge of socio-economic and political development based on the totality of all variables used in our study, but also based on other individual factors such as government efficiency, or GDP per capita. Our sample consists of 53 countries and 90 socio economic and political variables taken from various well known sources. Results for 1995 and 2011 are examined to check evolution through time. Important conclusions are: first, all socio-economic and political variables considered as totality and as groups grow in tandem; second each group of these four to five levels can separately generate the same classification of countries in terms of socio-economic development; third, some single indicators can be considered as good representatives of this development; and fourth, a 15 year span of development is not sufficient to change the classification of countries between the three main clusters of socio economic and political development.
\end{abstract}

Keywords: Institutions; Governance; Business; Political; Social; Economic; Culture; Development; Multidimensional scaling; Hierarchical clustering; Map

JEL classification: C38; Z13; Z19

\section{Introduction}

In this study we begin with the premise that economic development is a multidimensional phenomenon. Already 40 years ago, Baster [1] wrote that "...development is seen as multi-dimensional, involving changes in structure and capacity, as well as output..." Economic, social, political and other variables or indicators are important and indeed "...however important the economic dimension of development, it is dangerous to use it as a proxy for development" (ibid, p. 1). In addition, and as a corollary of the above premise we can regard development as an evolving system of all these various factors or variables that influence each other directly or indirectly [2]. Consequently we may infer that all these development factors are endogenous ${ }^{1}$ to the system and therefore we need multivariate tools of statistical analysis to examine such a system. Adelman and Morris [3] were the pioneers to use such tools as factor analysis and multidimensional scaling for the study of development. In particular, multidimensional scaling has been used by several scholars in economics related studies, including the seminal paper by Syrquin [4] in development, and more recently by Cinca et al. [5], Papalia and Bertarelli [6], and Akkucuk [7].

In this present study we endeavor to use multivariate analysis (by applying it to 89 variables and 53 countries) in order to bring some systematic quantitative evidence that economic development is a multidimensional, multi-variable phenomenon encompassing various factors originated in economic and social life, such as institutional, cultural, economic, governance, government and social. Since the 1970s, collection of data on all these factors has been methodical and rigorous and deserves to be rigorously analyzed in development studies. At the same time, in economics, the institutional and transactions economics as pioneered by three Nobel Prize Laureates, namely Coase,

${ }^{1}$ The endogeneity issue in economics has been emphasized since the 1980s. In the context of development see for example Rayp and Van De Sijpe (2007).
North and Williamson have introduced a strong interdisciplinary link between standard economics and parts of sociology or other social sciences. We will review the relevant literature in section 2 , but here we may summarize this link as follows: the economy has or needs a social embeddedness to develop and vice-versa, and this can take place through appropriate institutions, customs, culture, contracts and so on.

This link between economy and society has been the object of analysis in several knowledge fields and in particular in economics and sociology. For example from the evolutionary point of view, socioeconomic ${ }^{1}$ change can be taken as one combined force that includes entropic processes, technology, and evolutionary development (see for example, Chase [8]; Sanidas [9]. This link between economy and society also constitutes our composite hypothesis to test from a more quantitative perspective. Thus, more precisely, in this paper we will bring some relevant theories together and propose some partial hypotheses to test empirically. To test these hypotheses we will employ some multivariate techniques applied to data provided by the Institute for Management Development (IMD) and other wellknown databases such as Heritage Foundation, etc. These techniques are multidimensional scaling (MDS), and hierarchical clustering (HC). Note that these techniques are used in the context of economic development for the first time (to the best of our knowledge this is what our research in relevant literature has shown).

This field of research of providing multivariate quantitative

*Corresponding author: Elias Sanidas, Department of Economics, Seoul National University (SNU), Seoul, South Korea, Tel: 30 6970339027; E-mail: ellass@snu.ac.kr

Received November 30, 2017; Accepted December 13, 2017; Published December 15, 2017

Citation: Sanidas E (2017) Evidence of the Strong Nexus between Economic Social, Business, and Political Indicators across the World. Int J Econ Manag Sci 6: 481. doi: 10.4172/2162-6359.1000481

Copyright: @ 2017 Sanidas E. This is an open-access article distributed under the terms of the Creative Commons Attribution License, which permits unrestricted use, distribution, and reproduction in any medium, provided the original author and source are credited. 
evidence for links between society and economy is rather untapped as yet, and hence we hope that our empirical evidence is only a starting point for further research. In general, however, there have been some quantification attempts with pertinent analysis and results. Besides those already mentioned it is worth also mentioning Fedderke and Klitgaard [10] who examined a wide range of data regarding the relationship between economic growth and social indicators by using rank correlations and regressions of simultaneous equations; they emphasized the difficulties and dangers of using statistical techniques in quantifying this relationship and recommended amongst other things the following: "...The multicontextual and multivariate problems raised by the determination of economic growth deserve the attention of as wide a set of skills as is possible" (ibid, p. 484).

We will take up this last point as a prerequisite of our thesis in the present paper. Thus, overall, since development is multidimensional in character and since regression techniques might be inappropriate, we want to explore some multivariate techniques, as those ones already mentioned in the previous paragraph, in order to explore some particular aspects of economic growth and economic development based on a more precise theoretical model, that of Williamson's social analysis as explained in the next section. With his model we will be able to group this wide range of variables we have (89) and hence test the development phenomenon through the prism of his analysis. In this way our results will be more robust. The multivariate techniques used here are mainly based on dissimilarity distances between variables and thus do not offer a direct cause and effect explanation of the complex phenomenon of economic development; they only provide us with a spatial set of relationship both in terms of variables and countries in the sample. However, as already mentioned, since all variables are endogenous to each other (for example does GDP per capita growth cause computers per capita growth or vice versa?) we believe that multivariate analysis might be more adequate to examine development in several respects.

The particular aspects of economic development that we would like to explore here are the following ones expressed as questions. First, is there a pattern of socio-economic variables (related to institutions, governance, government, culture, social indicators, and economic development) that may follow more closely a theoretical model (hence a map may be constructed)? If, for instance, the probability of finding a variable anywhere on the map of all variables (and countries) is the same (in the contrary case this probability might be greater in a particular sub-area of the map), then there is no discernable pattern on the map, and so on. Second, do countries follow a pattern of development according to all these variables, for example according to high, middle, and low GDP per capita (or high, middle, and low level of government efficiency; and so on for some other variables)? Third, do all these categories of variables evolve together in time? If yes, then the patterns of development just mentioned should be the same regardless of the categories of variables we use (institutional, governance, etc). We will make some basic propositions to quantitatively assess or answer these questions in this paper.

\section{Theoretical Background}

Our background model $^{2}$ is the one proposed by the Nobel

'The term 'socio-economic' may have different meanings or names; for example for Castano (2007) it is called 'social capital' and it includes family, associations, State, etc. We will be more precise about our meaning in this paper as we progress into it. In any case it includes concepts from several social sciences, e.g. politics, and so on. laureate economist Williamson ${ }^{3}[11]$. According to this author (who complemented and added on North's [12] models of institutional factors affecting economic performance), economics of institutions are based on four distinct levels of social analysis. The first level or the lowest (or the most fundamental) in the social pyramid is the level of social embeddedness which comprises non calculative and spontaneous elements of informal institutions, customs, traditions, norms, and religion. All this we can call the underlying "culture" of society. It is at this first level where we have abundant sociological theoretical background. It comprises for example Granovetter's [13] embeddedness which is microanalytic and examines trust in terms of personal relations $s^{4}$. It also comprises studies by such scholars as Hofstede [14], Minkov and Hofstede [15], whose work on culture indexes is already well established (effectively we use these indexes in our paper). Table 1 contains the four variables of this level (indicated with the initial letter $C$, e.g. Cpdi is about "power distance index" or expectations of equal distribution of power in the society). For a good summary of the role of culture in economic development and the measurement of this role [16-18]. We must also emphasize that in the present paper we only included Hofstede's four composite culture indexes as representing Williamson's first level of "culture", but this does not mean that other culture variables, in a broader sense, do not exist in our analysis; for example our several variables related to freedom, ethical practices in business (Table 1), value system in society (Gvasy), national culture (Snacu) etc. are part of cultural values (but they are assigned in other categories such as institutional environment and social indicators in a more direct way). A more extensive use of cultural values such as those found in World Values Surveys is beyond the scope of the present study (in addition, they do not cover the same number of countries as in our study).

The second level is related to institutional environment $[19,20]$ where we have the formal rules of the game, especially property rules and by extension polity, judiciary and bureaucracy rules. Williamson calls this level the economics of property rights. Table 1 contains the variables of this level (indicated with the initial letter G). We assign variables from IMD and other sources into this broad category according to the following criteria: government initiated activities such as related to the just mentioned formal rules of the game (e.g. government efficiency, protection of personal security and private property, political stability, and so on); and institutional consequences of these initiatives such as education, technology, competition, corruption, parallel economy, health infrastructure, and so on (a good reference for the relationship between technology, institutions, and development is [21] for the effects of competition policy on development [22].

Level 3 or transaction cost economics, is related to governance or play of the game; it contains the elements of contracts and its purpose is to align governance structures with transactions. We can extend this level to encompass all factors that affect business governance and attitudes. Table 1 contains the variables of this level (indicated with the initial letter B). These B variables are directly related to "doing business" such as various types of freely conducting business, skills and training, internet and PC capabilities, labor relations, competent managers, technological capabilities, worker motivation, and so on; also, variables, such as public sector contracts open to foreign bidders

${ }^{3}$ This model is only a guiding model in our analysis and hence we do not aim at, strictly speaking, providing evidence to this model; rather we use this model as a guide to provide a general framework to our ideas and propositions.

${ }^{4}$ Williamson has been very prolific in his writings. Here we only refer to a very limited number of his papers or books: Williamson (1998a, 1998b, 2003, 2005, and 2008). 
Citation: Sanidas E (2017) Evidence of the Strong Nexus between Economic, Social, Business, and Political Indicators across the World. Int J Econ Manag Sci 6: 481. doi: 10.4172/2162-6359.1000481

Page 3 of 24

\begin{tabular}{|c|c|c|c|}
\hline 1 & Bcrma & S & Credibility of managers in society is stronTg (IMD WCY executive survey based on an index from 0 to 10 ) \\
\hline 2 & Bcusa & S & Customer satisfaction is emphasized in companies. (IMD WCY executive survey based on an index from 0 to 10) \\
\hline 3 & Bdete & S & Development and application of technology are supported by the legal environment. (IMD WCY executive survey based on an index from 0 to 10 ) \\
\hline 4 & Bemtr & S & Employee training is a high priority in companies (IMD WCY executive survey based on an index from 0 to 10 ) \\
\hline 5 & Bentr & S & Entrepreneurship of managers is widespread in business. (IMD WCY executive survey based on an index from 0 to 10 ) \\
\hline 6 & Betpr & S & Ethical practices are implemented in companies. (IMD WCY executive survey based on an index from 0 to 10 ) \\
\hline 7 & Bfisk & S & Finance skills are readily available (IMD WCY executive survey based on an index from 0 to 10 ) \\
\hline 8 & Bbfs & S & Banking and financial services do support business activities efficiently (IMD WCY executive survey based on an index from 0 to 10 ) \\
\hline 9 & Bfoin & S & Foreign investors are free to acquire control in domestic companies (IMD WCY executive survey based on an index from 0 to 10 ) \\
\hline 10 & Bfrbu & & $\begin{array}{l}\text { Business freedom is a quantitative measure of the ability to start, operate, and close a business that represents the overall burden of regulation as } \\
\text { well as the efficiency of government in the regulatory process. (Heritage) }\end{array}$ \\
\hline 11 & Bfrfi & & $\begin{array}{l}\text { Financial freedom is a measure of banking efficiency as well as a measure of independence from government control and interference in the } \\
\text { financial sector. (Heritage) }\end{array}$ \\
\hline 12 & Bfrin & & $\begin{array}{l}\text { Investment Freedom: In an economically free country, there would be no constraints on the flow of investment capital. Individuals and firms would } \\
\text { be allowed to move their resources into and out of specific activities both internally and across the country's borders without restriction. (Heritage) }\end{array}$ \\
\hline 13 & Bfrtr & & $\begin{array}{l}\text { Trade freedom is a composite measure of the absence of tariff and non-tariff barriers. The trade freedom score is based on two inputs: (i) the trade- } \\
\text { weighted average tariff rate, (ii) Non-tariff barriers (NTBs). (Heritage) }\end{array}$ \\
\hline 14 & Bfutd & S & Funding for technological development is readily available. (IMD WCY executive survey based on an index from 0 to 10 ) \\
\hline 15 & Bhepr & S & $\begin{array}{l}\text { Health problems (sicknesses, AIDS, alcohol, drug abuse, etc.) do not have a significant impact on companies. (IMD WCY executive survey based } \\
\text { on an index from } 0 \text { to 10) }\end{array}$ \\
\hline 16 & Binex & S & International experience of senior managers is generally significant (IMD WCY executive survey based on an index from 0 to 10 ) \\
\hline 17 & Binin & S & Investment incentives are attractive to foreign investors (IMD WCY executive survey based on an index from 0 to 10 ) \\
\hline 18 & Bintca & & Internet Capability: Number of internet users per 1000 people/Source: Computer Industry Almanac \\
\hline 19 & Bitsk & S & Information technology skills are readily available. (IMD WCY executive survey based on an index from 0 to 10 ) \\
\hline 20 & Blare & S & Labor relations are generally productive (IMD WCY executive survey based on an index from 0 to 10 ) \\
\hline 21 & Bpolp & S & Pollution problems do not seriously affect your economy. (IMD WCY executive survey based on an index from 0 to 10 ) \\
\hline 22 & Bpucf & S & Public sector contracts are sufficiently open to foreign bidders (IMD WCY executive survey based on an index from 0 to 10 ) \\
\hline 23 & Bquen & S & Qualified engineers are available in your labor market. (IMD WCY executive survey based on an index from 0 to 10 ) \\
\hline 24 & Bsema & S & Competent senior managers are readily available (IMD WCY executive survey based on an index from 0 to 10) \\
\hline 25 & Bshri & S & Shareholders' rights are sufficiently implemented. (IMD WCY executive survey based on an index from 0 to 10 ) \\
\hline 26 & Bskil & S & Skilled labor is readily available (IMD WCY executive survey based on an index from 0 to 10 ) \\
\hline 27 & Bsore & S & Social responsibility of business leaders is high (IMD WCY executive survey based on an index from 0 to 10) \\
\hline 28 & Bstma & S & Stock markets provide adequate financing to companies. (IMD WCY executive survey based on an index from 0 to 10 ) \\
\hline 29 & Bteca & & Indicator of Technological capabilities (Archibugi, D., and Coco, A., 2003), used for data of year 2000 \\
\hline 30 & Bteco & S & Technological cooperation between companies is developed (IMD WCY executive survey based on an index from 0 to 10 ) \\
\hline 31 & Bwomo & S & Worker motivation in companies is high (IMD WCY executive survey based on an index from 0 to 10) \\
\hline 32 & Cindi & & $\begin{array}{l}\text { Individualism (IDV): On the individualist side we find societies in which the ties between individuals are loose: everyone is expected to look after } \\
\text { him/herself and his/her immediate family. (Hofstede's scores) }\end{array}$ \\
\hline 33 & Cmasc & & $\begin{array}{l}\text { Masculinity (MAS) refers to the distribution of roles between the genders. Men's values contain a dimension from very assertive and competitive to } \\
\text { modest and caring. The assertive pole has been called 'masculine' and the modest, caring pole 'feminine'. (Hofstede's scores) }\end{array}$ \\
\hline 34 & Cpdi & & $\begin{array}{l}\text { Power Distance Index (PDI) is the extent to which the less powerful members of organizations and institutions accept and expect that power is } \\
\text { distributed unequally. This represents inequality, defined from below. It suggests that a society's level of inequality is endorsed by the followers as } \\
\text { much as by the leaders. (Hofstede's scores) }\end{array}$ \\
\hline 35 & Cunav & & $\begin{array}{l}\text { Uncertainty Avoidance Index (UAI) deals with a society's tolerance for uncertainty and ambiguity; Uncertainty avoiding cultures try to minimize the } \\
\text { possibility of unstructured situations - novel, unknown, surprising, different from usual-by strict laws and rules, safety and security measures and on } \\
\text { the philosophical and religious level by a belief in absolute Truth. People in uncertainty avoiding countries are also more emotional, and motivated } \\
\text { by inner nervous energy. (Hofstede's scores) }\end{array}$ \\
\hline 36 & Ecoli & & Cost of Living: index of a basket of goods \& services in major cities, including housing (New York City=100)(IMD) \\
\hline 37 & Eempp & & Employment, Percentage of population (IMD) \\
\hline 38 & Eexind & & Exports breakdown by economic sector/Industry, Percentage of total exports (IMD) \\
\hline 39 & Efrfc & & $\begin{array}{l}\text { Fiscal freedom is a measure of the tax burden imposed by government. The fiscal freedom component is composed of three quantitative factors: (i) } \\
\text { the top tax rate on individual income, (ii) the top tax rate on corporate income and (iii) Total tax revenue as a percentage of GDP. (Heritage) }\end{array}$ \\
\hline 40 & Efrmo & & $\begin{array}{l}\text { Monetary freedom combines a measure of price stability with an assessment of price controls. Both inflation and price controls distort market } \\
\text { activity. Price stability without microeconomic intervention is the ideal state for the free market (Heritage) }\end{array}$ \\
\hline 41 & Egdppc & & GDP per capita, PPP (current international \$) (World Bank data) \\
\hline 42 & Egocp & & Government consumption expenditure Percentage of GDP (IMD) \\
\hline 43 & Ehocp & & Household consumption expenditure Percentage of GDP (IMD) \\
\hline 44 & Eindp & & Economic sectors/Industry, Breakdown as a percentage of GDP (IMD) \\
\hline 45 & Einvp & & (Investment) Gross fixed capital formation Percentage of GDP (IMD) \\
\hline 46 & Elapr & & Labor productivity (PPP) (IMD) \\
\hline 47 & Eovpr & & Overall productivity (PPP) (IMD) \\
\hline 48 & Esavp & & Gross domestic savings Percentage of GDP (IMD) \\
\hline 49 & Gadpo & S & Adaptability of government policy to changes in the economy is high (IMD WCY executive survey based on an index from 0 to 10 ) \\
\hline
\end{tabular}


50 Gbrdr S $\quad$ Brain drain (well-educated and skilled people) does not hinder competitiveness in your economy (IMD WCY executive survey based on an index from 0 to 10 )

51 Gbrib S Bribing and corruption do not exist (IMD WCY executive survey based on an index from 0 to 10)

52 Gbure S $\quad$ Bureaucracy does not hinder business activity (IMD WCY executive survey based on an index from 0 to 10)

53 Gcole S Competition legislation is efficient in preventing unfair competition (IMD WCY executive survey based on an index from 0 to 10)

54 Gcorc Control of Corruption captures perceptions of the extent to which public power is exercised for private gain, including both petty and grand forms of corruption, as well as "capture" of the state by elites and private interests. (WGI project)

55 Gdeci S Government decisions are effectively implemented (IMD WCY executive survey based on an index from 0 to 10)

56 Gdiin $\quad$ S $\quad$ Distribution infrastructure of goods and services is generally efficient (IMD WCY executive survey based on an index from 0 to 10)

57 Gedsy $\mathrm{S}$ Educational system meets the needs of a competitive economy. (IMD WCY executive survey based on an index from 0 to 10)

58 Genla $\quad$ S $\quad$ Environmental laws and compliance do not hinder the competitiveness of businesses. (IMD WCY executive survey based on an index from 0 to 10)

59 Geqop S Equal opportunity legislation in your country encourages economic development (IMD WCY executive survey based on an index from 0 to 10)

60 Gfrcor $\quad$ Freedom from Corruption - Corruption erodes economic freedom by introducing insecurity and uncertainty into economic relationships. The higher the level of corruption, the lower the level of overall economic freedom and the lower a country's score. (Heritage)

61 Ggoef Government Effectiveness captures perceptions of the quality of public services, the quality of the civil service and the degree of its independence from political pressures, the quality of policy formulation and implementation, and the credibility of the government's commitment to such policies. (WGI project)

62 Ghein S Health infrastructure meets the needs of society. (IMD WCY executive survey based on an index from 0 to 10)

63 Gimla $S$ Immigration laws do not prevent your company from employing foreign labor (IMD WCY executive survey based on an index from 0 to 10)

64 Ginst S $\quad$ The risk of political instability is very low (IMD WCY executive survey based on an index from 0 to 10)

65 Gipr S Intellectual property rights are adequately enforced. (IMD WCY executive survey based on an index from 0 to 10)

66 Gjust S Justice is fairly administered. (IMD WCY executive survey based on an index from 0 to 10)

67 Glare S Labor regulations (hiring/firing practices, minimum wages, etc.) do not hinder business activities (IMD WCY executive survey based on an index from 0 to 10 )

68 Glawr $\quad$ Rule of Law captures perceptions of the extent to which agents have confidence in and abide by the rules of society, and in particular the quality of contract enforcement, property rights, the police, and the courts, as well as the likelihood of crime and violence. (WGI project)

69 Gmaint S Maintenance and development of infrastructure are adequately planned and financed. (IMD WCY executive survey based on an index from 0 to 10)

70 Gpaec S Parallel (black-market, unrecorded) economy does not impair economic development (IMD WCY executive survey based on an index from 0 to 10)

71 Gpese S $\quad$ Personal security and private property are adequately protected (IMD WCY executive survey based on an index from 0 to 10)

72 Gpolst Political Stability and Absence of Violence/Terrorism captures perceptions of the likelihood that the government will be destabilized or overthrown by unconstitutional or violent means, including politically-motivated violence and terrorism. (WGI project)

73 Gpri $\quad$ Property rights component is an assessment of the ability of individuals to accumulate private property, secured by clear laws that are fully enforced by the state. (Heritage)

74 Grequ Regulatory Quality captures perceptions of the ability of the government to formulate and implement sound policies and regulations that permit and promote private sector development (WGI project)

75 Gtrapo S $\quad$ Transparency of government policy is satisfactory (IMD WCY executive survey based on an index from 0 to 10)

76 Gtrcor (Transparency) Corruption Perceptions Index (CPI) orders the countries of the world according to the degree to which corruption is perceived to exist among public officials and politicians. (Transparency International)

77 Guned S University education meets the needs of a competitive economy. (IMD WCY executive survey based on an index from 0 to 10)

78 Gunle S Unemployment legislation provides an incentive to look for work (IMD WCY executive survey based on an index from 0 to 10)

79 Gvasy S Value system in your society supports competitiveness. (IMD WCY executive survey based on an index from 0 to 10)

80 Gvoac Voice and Accountability captures perceptions of the extent to which a country's citizens are able to participate in selecting their government, as well as freedom of expression, freedom of association, and a free media (WGI project)

81 Sdera $\quad$ Dependency Rate: Population under 15 and over 64 years old, divided by active population (15 to 64 years) (IMD)

82 Sefri Economic freedom index is designed to measure the consistency of a nation's institutions and policies with economic freedom. The key ingredients of economic freedom are: (1) personal choice, (2) voluntary exchange coordinated by markets, (3) freedom to enter and compete in markets, and (4) protection of persons and their property from aggression by others (Cato)

83 Sflad S $\quad$ Flexibility and adaptability of people are high when faced with new challenges. (IMD WCY executive survey based on an index from 0 to 10 )

84 Sfret Overall Score of economic freedom: The highest form of economic freedom provides an absolute right of property ownership, fully realized freedoms of movement for labor, capital, and goods, and an absolute absence of coercion or constraint of economic liberty beyond the extent necessary for citizens to protect and maintain liberty itself. (Heritage)

85 Sgci Global Competitiveness Index measures the set of institutions, policies, and factors that set the sustainable current and medium-term levels of economic prosperity (WEF)

86 Shdi HDI: Combines economic - social - educational indicators/Source: World Bank, Human Development Report

87 Shele Healthy life expectancy: average estimate (IMD)

88 Snacu S National culture is open to foreign ideas. (IMD WCY executive survey based on an index from 0 to 10)

89 Squli S Quality of life is high (IMD WCY executive survey based on an index from 0 to 10)

90 Surpo $\quad$ Urban population, Percentage of total population (IMD)

Notes: (i) Unless otherwise indicated, the source of the variables is IMD; (ii) The (s) whenever indicated for a variable means data are based on Annual Executive Online Survey. (iii) WGI project stands for (Worldwide Governance Indicators project), WEF (World Economic Forum), UNDP (United Nations Development Program), Transparency International, Cato (Cato Institute), Heritage (Heritage Foundation), Hofstede's scores.

Table 1:Information on socioeconomic variables. 
(Bpucf) which even though it is governance oriented, it directly affects business operations. Note that although we took much care to assign all these variables into the groups $\mathrm{G}$ or $\mathrm{B}$ according to the above general criteria, we cannot expect our classification to be $100 \%$ correct. However, even if it is not $100 \%$ correct, we can still discuss about it and change classification once we examine the maps of dissimilarity matrices as will be explained further below. This same remark is applicable to all other groups of variables as defined in this section.

Finally the fourth and higher level in the hierarchy of social analysis is what Williamson calls the neo-classical economics and agency theory that comprises the elements of resource allocation, employment, prices, and quantities. Table 1 contains the variables of this level (indicated with the initial letter $\mathrm{E}$ ). We chose them because these economic variables are deemed to represent the main aspects of an economy: GDP per capita, consumption expenditure as percent of GDP, productivity measures, employment as percent of population, exports by industry, and so on as per usual practice. These economic variables are also variables of growth models or extended growth and development models such as those suggested by Landau [23], Knowles and Garces-Ozanne [24], and Norton [25].

As we go from the first level to the fourth level it takes shorter and shorter time to make changes in society. Thus, the first level of culture might take even 100 to 1000 years for changes to take place, the second level 10 to 100 years, the third level 1 to 10 years, and the fourth level is continuous all the time [11]. We should note that the levels 2 and 3 of Williamson's analysis can also be considered to be social indicators. This is clearly suggested, for example, by the Global Reporting Initiative (GRI) launched in 1997 and whose guidelines are a framework for reporting on an organization's economic, social and environmental performance [26]. In addition, we should note that sociologists such as Granovetter [13] and Lewis [27] criticize transaction costs economics as being too restricted in the sense that social institutions are part of a wider open social system and hence do not depend on transaction costs solely. Furthermore, we will include in our model here a fifth group (not explicitly mentioned by Williamson) which takes into account several social indicators (in a broad sense). The variables of this group (indicated with the initial letter S) are shown in Table 1 and come from various sources. 5 . Some of these $S$ variables are closely related to business activities such as "Sefri" (economic freedom), or related to a combination of institutional and other factors such as "Sgci" (global competitiveness index).

Williamson's basic social analysis model just briefly described addresses mainly one issue: the categorization of all socio-economic factors into four main levels or groups. However, it does not tell us directly how these four groups evolve or grow through time except that it takes longer for culture to change, and so on as seen above. Consequently we need to complement Williamson's model with some other elements in order to theoretically assert that all levels have some common ground of parallel or in tandem development. Nonetheless, we will resolve this theoretical issue mainly in a quantitative way. Meanwhile let us mention some extra related theoretical points. First, is an economy embedded in social relations or vice-versa? According to Polanyi [28], social relations might be embedded in the economic system. It is worth reproducing here Polanyi's view as quoted in Jessop [29].

${ }^{5}$ Initially we also included two crime indicators (homicide and robbery rates); however we subsequently excluded them from our current analysis because of their extreme cases (such as very high homicide in some countries) which made the MDS map difficult to visualize.
Instead of economy being embedded in social relations, social relations are embedded in the economic system. The vital importance of the economic factor to the existence of society precludes any other result. For once the economic system is organized in separate institutions, based on specific motives and conferring a special status, society must be shaped in such a manner as to allow that system to function according to its own laws. This is the meaning of the familiar assertion that a market economy can function only in a market society.

Polanyi's just quoted passage is important in our paper. In particular, the conclusion in this quote that "a market economy can function only in a market society" might have the following implication: the more an economy has existed as a market economy the more it has built an appropriate set of institutions that support this market economy (because it becomes more of a market society). This might also infer that a well-developed market society has a welldeveloped market economy and hence it has a strong economy. A good example of all this is the recent Chinese society and economy. When appropriate institutions were implemented during the 1980s, this country became a market society (thus it left behind the communist non-market society) and developed a strong market economy. What made the transition from a non-market economy to a market economy so successful? The answer is the introduction of appropriate rules and institutions that transformed the Chinese society into a market society. We want to bring some evidence that across several countries we obtain the same result: market society (institutions, governance and culture) and market economy evolve in tandem.

Second, what is the intrinsic relationship between economic development and institutions? Initially, we note that Williamson in his 1998 b paper (p. 75) emphasized that, "the study of economic institutions needs to make provision for two background conditions: the condition of social embeddedness (to which sociologists refer)... and the attributes of human actors ${ }^{6} . . . "$ As we have already seen above, his model contains four levels of analysis; in particular, his levels 2 and 3 of institutions and governance are important in determining the "economizing" aspect of economies. Other writers such as Dixit [30], Kunneke [31], Key et al. [32], and so on agree with this type of analysis ${ }^{7}$. Then, according to another Nobel Laureate's work, namely that of North, the historical evolution of institutions and economic development grow in tandem. As summarized by Boliari [33], Yeager [34] proposed the following schemas of North's model:

(i) The static case where technology is kept constant: institutions $\rightarrow$ transaction costs $\rightarrow$ creation of markets $\rightarrow$ specialization and division of labor $\rightarrow$ productivity $\rightarrow$ economic performance.

(ii) The dynamic case where technology advances: institutions $\rightarrow$ behavior of organizations $\rightarrow$ process of creative destruction $\rightarrow$ technological progress $\rightarrow$ economic wealth.

In summary, for the remaining of this paper we will accept that there are four (or five) distinct ${ }^{8}$ categories of factors that influence a society's evolution through time and space in the world. Thus, a society's institutional environment (one distinct category), its business governance and contract foundations (another distinct category)

${ }^{6} \mathrm{He}$ refers to psychology.

It is also appropriate to distinguish between private types of governance and contracts (e.g. through vertical integration) and state governance and contracts as Williamson (2005) has already done. This distinction does not affect our analysis, but on the contrary it re-enforces it.

${ }^{8}$ The emphasis is on the 'distinct' nature of these four categories. Here "distinct" means that variables within each such category are more closely related between themselves than between them and other variables of the other categories. 
are interacting in a significant way in order to enhance economic development (another distinct category). All these three categories are in addition interacting with culture and slow changing informal rules and conventions. In particular business governance and organizational structure is closely linked with institutional framework, market conditions, transaction costs, and uncertainty. According to this brief theoretical background we then propose to test empirically the following 3 propositions:

\section{Proposition 1}

The four categories of institutional environment, business governance, economic development, and culture (based on Williamson's model) form four distinct categories that we should be able to distinguish on an appropriate map empirically constructed which would contain all variables (89 for 2011) pertinent to the four categories. We will also check for the inclusion of social indicators as a fifth category.

\section{Proposition 2}

From these variables (pertinent to the four or five categories) we can infer an overall map of socio-economic development for the countries examined in our sample (53). This country development map would well represent our expectations we have about three broad categories of development (high, medium, and low). Alternatively we propose that some well-known indicators such as GDP per capita or government efficiency can adequately describe these three groups of development.

\section{Proposition 3}

If both propositions 1 and 2 are true, then overall institutional and business variables grow in tandem with economic, cultural and human or social variables (all variables considered together and not individually.

\section{Data and Methodology}

We must say from the outset that development factors or indicators are only an approximation of what we try to measure [35]. Nonetheless, there has been a substantial progress in collecting appropriate and relevant data. We will mostly use data from Institute for Management Development (IMD). This publication publishes data on many categories of socio-economic ${ }^{9}$ factors. Some of them are official secondary data such as employment, as compiled in other sources of data such as World Bank, IMF, and so on. Some of them are, however, data based on surveys regularly conducted by IMD. In our paper we will use both types of data, with the proviso that the reader be aware of the surveys' validity involved in using some of these factors, especially when comparing data in two different points of time as we also do here. Nevertheless, the use of other sources for similar variables and their overall mutual comparison (as will be shown further below) makes their use more pertinent. Thus, from the IMD publications we include most variables on business and government efficiency. From the Worldwide Governance ${ }^{10}$ Indicators ${ }^{11}$ (WGI) Project Report, we obtained more variables (six) on government efficiency and political stability. From the Global Competitiveness Report of World Economic Forum (WEF), we obtained the variable "global competitiveness index" (Sgci). From Heritage Foundation we obtained variables (nine) mainly

'The term 'socio-economic' is used here in the general sense of pertaining to both society and economy as already analyzed in the previous two sections.

${ }^{10}$ In our paper we use the term governance to indicate business governance issues. ${ }^{11}$ Recently these indicators have been scrutinized for their validity and relevance to development (see for example Langbein and Knack, 2010). linked to freedom-related issues in business. From Cato Institute we obtained one extra variable that measures economic freedom overall (Sefri). From Transparancy International we used one extra variable on corruption issues (Gtrcor). Finally from the Hofstede's Cultural Dimensions Report, we obtained variables on culture. Note that all these sources of socio-institutional and business variables are used to cross check the validity of these sources of data; thus the corruption index of IMD will be checked against other corruption variables from other sources, and so on. Also from IMD we only include a limited number of economic variables that adequately represent national economies at a given point of time (as already explained). Table 1 lists all variables used in our study; it also indicates if they are survey related and the source of each variable. In total there are $32 \mathrm{G}, 30 \mathrm{~B}, 13 \mathrm{E}, 10$ $\mathrm{S}$, and $4 \mathrm{C}$ variables (for 2000 there is one extra $\mathrm{B}$ ). Also note that for all these variables, the scores are in descending order of importance (thus the country with the best score for the " $\mathrm{x}$ " variable has the highest score); nonetheless, even if the order is reversed for some variables the Euclidean distances do not change.

The same Table 1 also shows our a priori categorization of all these variables according to Williamson's model as described in the previous section. Thus, the first category is institutional environment which includes government intervention, political stability, education and infrastructure (a good survey related to development and infrastructure is Straub, 2011); these variables are indicated with the initial capital letter of ' $G$ ' in front of the acronym. The second category is governance on the micro level, hence contracts and attitudes of firms managers and employees; these variables are indicated with the initial capital letter of ' $\mathrm{B}$ '. The third category is economic variables that represent the level of economic development at a given point of time; these variables include the main aspects of how the economy is structured in terms of employment, investment, exports, GDP per capita, and so on; these variables are indicated with the initial capital letter of ' $E$ '. The fourth category is culture factors that show a long process of informal rules, attitudes, customs, and so on; these variables are indicated with the initial capital letter of ' $C$ '. Finally, although not mentioned in our four categories of Williamson's model, we include another type of data called 'social' variables which are micro indicators of social standards such as 'human development index', 'healthy life expectancy', and so on; these variables are indicated with the initial capital letter of ' $S$ ' in Table 1. These social variables could be considered as part of any one of the previous four categories at the same time. Thus, only empirically we can uncover where exactly these variables "S" are located. Furthermore, once we know about their location, we can relate it to Williamson's model. All this will be discussed in the next section.

We must now briefly comment on the validity of using all these data coming from different sources; being either secondary or survey data; and used at a given point of time, hence being cross sectional in nature. We believe that these data, secondary and survey, are complementing each other as used in the multivariate techniques of MDS, and HC because we are not concerned with cause and effect issues. Also, using several sources of data may provide us the opportunity to check the validity of these sources according to the multivariate techniques. Finally, a cross section of data is used at two different points of time (1995 and $2011^{12}$ ), thus making a comparison between these years possible. Furthermore, the variables compiled at a given point of time, e.g. 2011, show the level of achievement of each country up to that point (e.g. 2011) for each one of the variables, thus inferring whether

${ }^{2}$ We also examined data for 2000 and 2008 but the results are not reported here as they do not change our conclusions. 
these various variables grow in tandem or not. In summary, we include 89 (or 90 for 2000) variables chosen from the database of IMD (67), plus some extra variables (22) from other data bases (Table 1) and for the years 1995 and 2011. The number of countries 53 is chosen because the IMD and the other sources of data have consistently observations through time for these countries only ${ }^{13}$.

In terms of methodology, we first want to show how all these socio-economic variables (considered in totality or in groups) are put on a map so that we can observe if there is a spontaneous grouping into the categories we suggested in the previous section according to Williamson's model. We also want to further check this grouping of socio-economic variables by examining its image (reflexion) into a grouping of countries: is there any spontaneous grouping of countries into developed, less developed and developing nations? What we do not want to do in our paper (mainly for space reasons as this would require another full paper) is to directly detect any causality or dependence mechanisms between economic development and various factors (hence in terms of methodology we will not use any regression techniques). On the contrary, for our purposes we use $\mathrm{s}^{14}$ mainly two multivariate complementary methods, those of MDS and HC.

We turn now into methodological issues. For map construction we use MDS, a method widely used in social sciences. For a basic manual on MDS see Kruskal and Wish [36]; for a general treatment of MDS in social sciences see Hair et al. [37]; for applications of MDS see for example Craig et al. [38]; Cinca et al. [5]; for a modern treatment of MDS see Borg and Groenen [39]. With this method we can calculate relative mutual distances between variables according to simple principles of distance measurement and standardization. Thus, for the latter we chose the transformation of all variables into the standardized form 0 to $1^{15}$ so that differences in the size of numbers do not influence calculated distances. One ${ }^{16}$ type of such distances is used here (as in most research articles), namely the Euclidean distance which is the standard straight line distance between two points (as per Pythagoras's formula). For a simple way to visually see the working of MDS, Table 2 shows the similarity ${ }^{17}$ data for four ${ }^{18}$ variables, and Figure 1 shows how these four variables are depicted on a straight line according to their pair wise distances if we consider only one dimension. In this particular example, we can see that the relative similarities can be perfectly represented by relative distances as seen in Figure 1.

${ }^{13}$ In this sample of 53 countries we have a well-balanced mixture of countries at various stages of development. Hence we do not think the selection bias is important here.

${ }^{14}$ There are other methods to use in order to detect relationships between governance, economic performance, and other categories. For example Agarwal and Samanta (2006) use correlation techniques to measure relationships between indexes of governance, social progress etc. Our methodology is more indirect and makes use of multivariate rather than univariate analysis.

${ }^{15}$ The exact formula for this transformation is $z_{\mathrm{i}}=y_{\mathrm{i}}$-lower bound of $\mathrm{y}$

upper bound of $\mathrm{y}$-lower bound of $\mathrm{y}$ paper because of the following two reasons: i) most variables (those based on surveys and social indicators) are already calculated based on the 0 to 1 scale; ii) other standardization measures such as the z-scores are open sets (minus infinity to plus infinity) or they emphasize the mean or standard deviation, and so on.

${ }^{16}$ Other types of distances such as the 'block' or Chebychev ones yield similar results to those obtained with the Euclidean distance. In addition these other types might be less adequate to use in our paper; for example, the Chebychev distance is defined as the greatest difference across all variables in their clusters.

${ }^{17}$ One refers to similarity if a survey has established similarity of for example a particular product in terms of one or more characteristics for the product. This similarity is then measured as the least distance (e.g. Euclidean) between these characteristics.

${ }^{18}$ For more than four variables the task is much more difficult to show (see Hair et al, 2006, for a similar example).

\begin{tabular}{|c|c|c|c|c|}
\hline Variables & A & B & C & D \\
\hline A & - & 1 & 12 & 3 \\
\hline B & & - & 9 & 5 \\
\hline C & & & - & 7 \\
\hline D & & & & - \\
\hline
\end{tabular}

Note: Lower values indicate greater similarity, with 1 being the most similar pair and 12 the least similar pair.

Table 2: Similarity data (rank orders) for pairs.

A two or three dimensional representation of the same data is also possible; however this representation might not be as perfect in two dimensions as it is in one dimension for our example data. In our paper, further below, we show for our real data both the one dimension and two dimensions maps for our initial case of data so that the reader can clearly see how MDS works on our data. Usually, one dimension is insufficient (this can be checked with the stress test), whereas most often two dimensions represent original (dis)similarities better (thus with a higher R square). We must also note that we follow here the current terminology regarding the words similarity versus dissimilarity; thus we refer to a "dissimilarity" matrix if distances are measured, whereas if correlations are measured between variables then we refer to a "similarity" matrix. This distinction is important because MDS uses distances and hence their corresponding dissimilarity matrix is used in order to represent data with maps. However, as we will mention in the next section, our dissimilarity matrix is very similar to a similarity matrix which is based on correlation coefficients. This indicates that usually (not always) small distances can be interpreted with high correlation coefficients.

Besides MDS we also use hierarchical clustering (HC) which creates clusters of variables according to distances in a hierarchical way (or like a tree structure). As a result, countries (53 in our case) have "preferences" or "performances" in terms of each one of the 89 variables. Then the latter are put into major and minor groups according to their mutual dissimilarities (distances) or similarities (correlations). With our type of data MDS and HC are very similar in nature and should support each other in terms of their results. The HC provides some extra possibilities in terms of correlation measures and of course in terms of clustering (e.g. between or within groups, complete linkage, etc) ${ }^{19}$. Also, we could have referred to a better known multivariate technique, that is, discriminant analysis, but unfortunately lack of data does not allow us to fully explore the possibilities of this technique (the number of countries should be about 5 times the number of variables). Finally, dissimilarity matrix analysis, a rather simple analysis but important, will be briefly used further below. These methods are complementing each other; however, we put more emphasis on DMS as this method is the only one to show maps, thus easy to visualize countries or variables in relation to each other.

\section{Empirical Results}

\section{Clusters of economic development for 89 variables}

We will first present our MDS map for our basic data matrix (53 countries and 89 variables for 2011) by considering one dimension only (that is, on a straight line, see Figure 2). We can thus see that almost all B group variables are lined one after the other at the upper part of the straight line, and the $G$ group variables at the lower part of it (with some exceptions). This is a first strong indication that the

${ }^{19}$ It is beyond the scope of this paper to provide an extensive comparison between MDS and HC (see for instance Hair et al, 2006; Borg and Groenen, 2010). For HC for more details, see Hair et al (2006). 
Citation: Sanidas E (2017) Evidence of the Strong Nexus between Economic, Social, Business, and Political Indicators across the World. Int J Econ Manag Sci 6: 481. doi: 10.4172/2162-6359.1000481

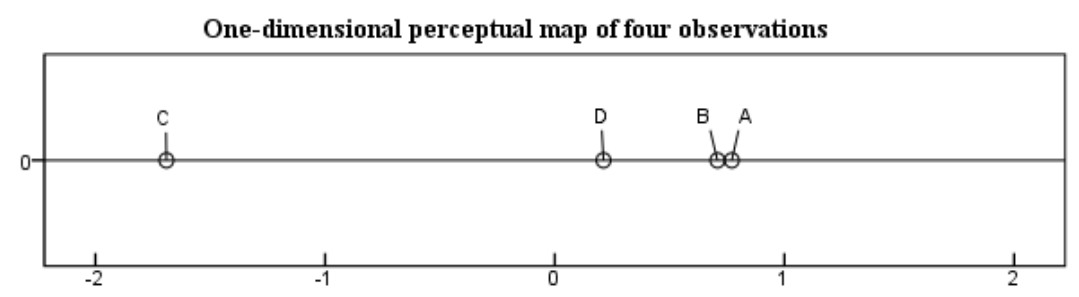

Notes: This map is based on the distances shown in Table 2. The only pair which does not match the similarity numbers of Table 2 is AD versus BD: on this figure, $A D>B D$, but in the original data of Table $1, A D<B D$.

Figure 1: One dimensional map.

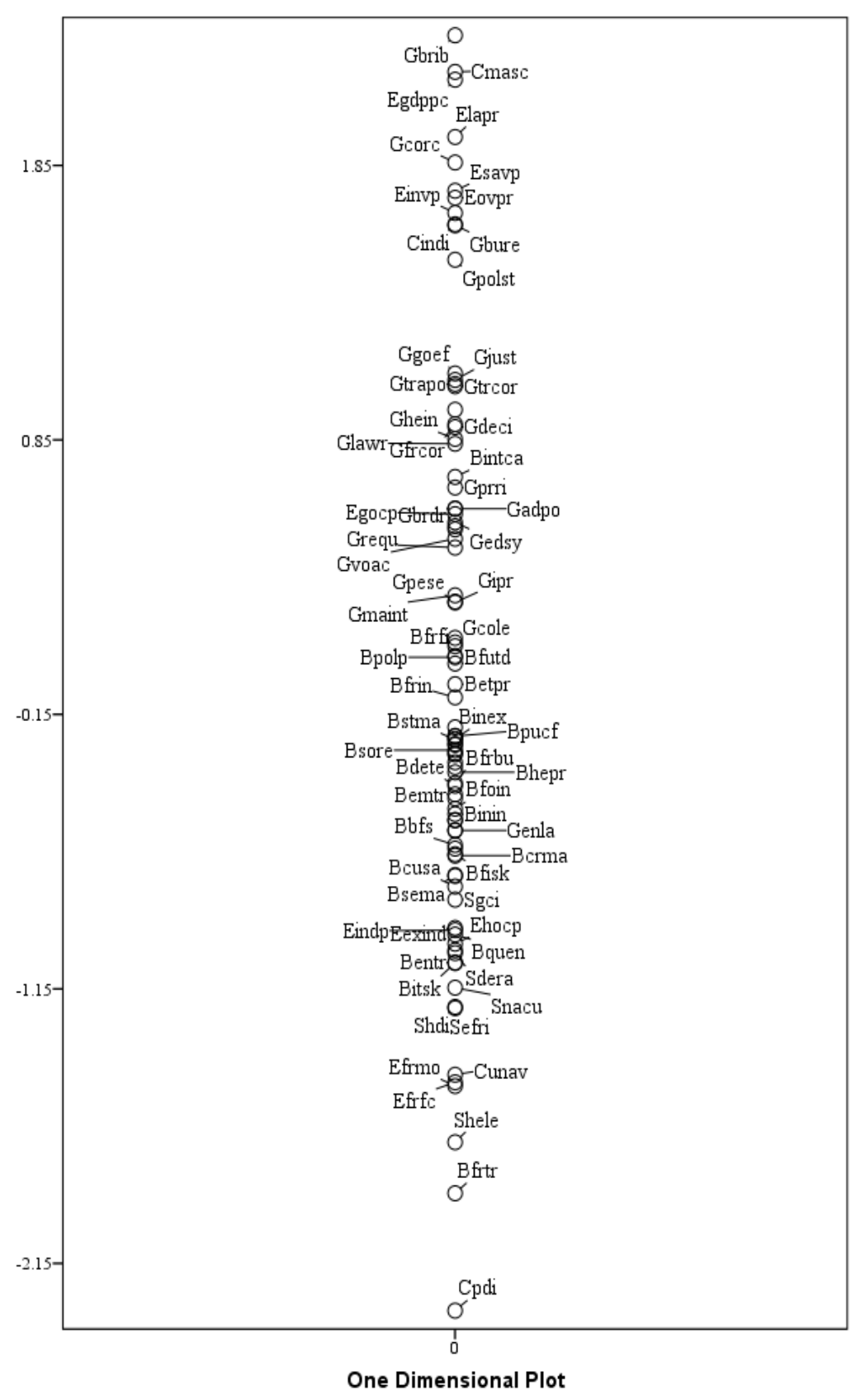

Notes: Euclidean distance, 0 to 1 standardization, Stress $=0.29, \mathrm{R}^{2}=0.77$.

Figure 2: 2011 map of 89 socio-economic indicators for 53 countries. 
G and B groups are rather separate and form two important clusters. As expected the two statistical tests are not satisfactory. The stress test indicates that a two dimensional map should be preferred and the $\mathrm{R}$ square is rather average. Consequently we now present the two dimensional map. Figure 3 shows the results with Euclidean distance (similar results are obtained with Chebychev distances ${ }^{20}$ ). In Figure 3 we can see that the groupings based on their mutual distance make reasonable sense and agree with our expectations. In particular, first the institutional governance (or level 2 of 'institutional environment' to use Williamson's terminology) factors (shown with the letter $\mathrm{G}$ in front of each variable in Figure 3) are located together on the west part of the $\mathrm{map}^{21}$, and towards the north. Both the IMD indicators and those based on other sources are in agreement.

The further to the east we go on the map in Figure 3, the more closely we get to business (firm or micro level) governance. At the boundary of the two levels of governance we find variables that depend on institutional governance and have a direct effect on infrastructure and technology, such as 'funding for technology development' (Bfutd), and 'intellectual property rights' (Gipr). This is supported by studies such as that by Kunneke [31] who provided evidence of the positive interrelations between technological and institutional changes. Also, most educational factors such as 'university education' (Guned) that depend on institutional governance are located near or at the boundary between the two types of governance. Thus, overall, we can see that between and within the two broad categories of institutional environment and business governance, we have educational, technological and similar types of variables. All these variables and categories are also conventionally called 'multi-level governance' from a sociological point of view [40].

Second, as already hinted at in the previous paragraph, the business contracts or governance (level 3 of Williamson's model) variables (shown with the letter B in front of each variable in Figure 3) are situated on the east part of the map. There we can discern some particular tendencies; for example, the further south we look at (especially below the $\mathrm{x}$-axis) variables are more related to the factor of production "labor". Thus we have the variables 'unemployment legislation' (Gunle) and 'immigration laws' (Gimla) as seen through the eyes of surveyed business executives; we also have human capital variables such as 'skilled labor availability' (Bskil) and 'credibility of managers' (Bcrma); or 'national culture' (Snacu), which again as seen through the eyes of surveyed executives indicate how these attitudes might influence business and society; and so on [41-43].

We should also emphasize that for the variables of levels 2 and 3 of Williamson's model we used several sources (such as IMD, Heritage, WGI project, etc); thus variables from these various sources are located in similar locations on the map hence confirming their mutual and own validity (similar variables from different sources are thus cross checked). For example, Gbrib, Gcorc, and Ggoef are all very closely located on the map (these three variables are from two different sources). Also, the extra technological variable Bteca (an indicator of technological capabilities for the 2000 map only) agrees with the IMD technology variables (e.g. Bteco) in terms of their position in the map (the map for 2000 is not included here for space limitations). Another example is the discernible sub-group of "freedom" variables (Table 1 for their names and acronyms) which is mostly situated on the north east part of the map for $2011[44,45]$.

Third, most of economic (level 4 of Williamson's model) variables (shown with the letter $\mathrm{E}$ in front of each variable in Figure 3 ) are located on the lower south side of the map; they are scattered around in that area. However, some of them are situated closer to the previous two

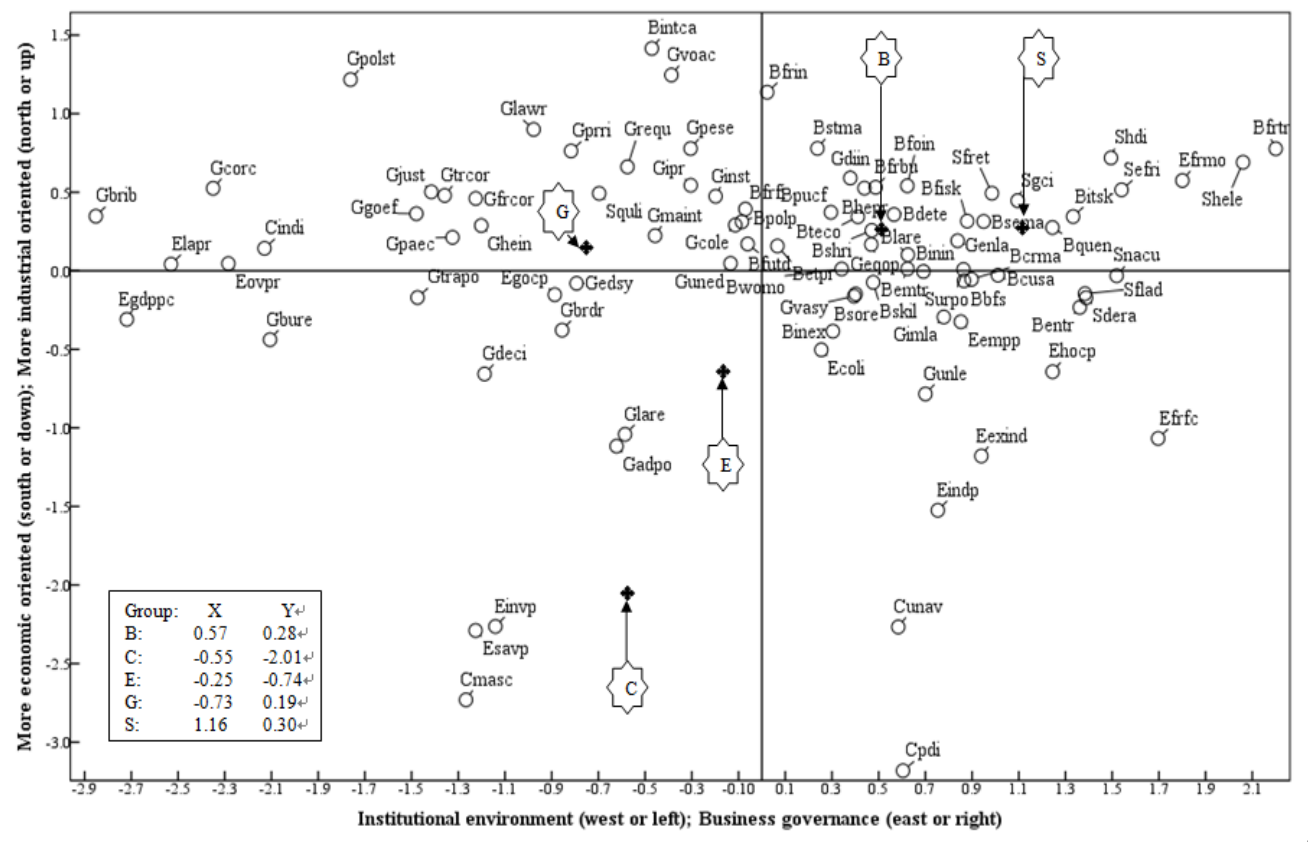

Notes: Euclidean distance, 0 to 1 standardization, Stress $=0.13, R^{2}=0.94$.

Figure 3: 2011 map in terms of 89 variables (as per 53 countries).

${ }^{20}$ They are available on request.

${ }^{21}$ This also means that all these factors which are closely located on the MDS map have a high probability to be also highly positively correlated with each other. major institutional and governance categories. Thus, a couple of them -'cost of living' (E. coli) and 'employment as a percentage of population 
(Eempp) are situated within the group of business governance thus indicating their close relationship with the latter; this is not an unexpected result as these economic variables are important in a more direct way to business operations [46-48]. The variable 'government consumption expenditure' (Egocp) is located very close to the institutional and educational variables on the left side of the map and very close to the $\mathrm{x}$-axis. In addition, as already mentioned above, three economic variables of macroeconomic efficiency (Egdppc, Elapr, and Eovpr) are located on the far west side of the map together with some institutional efficiency variables ${ }^{22}$. When we eliminated all economic variables and all culture variables from the MDS map, the distribution of the remaining (over 70) institutional and business variables still showed a similar configuration of mutual distances as seen in Figure 3 (graph not shown here). Similar results were also obtained when only the culture variables were eliminated $[49,50]$.

Fourth, three out of our four "pure" culture (level 1 of Williamson's model) variables (shown with the letter $\mathrm{C}$ in front of each variable in Figure 3) are located on the far south of the map; and one, the 'individualism' (or Cindi as acronym) is located in the midst of (or close to) institutional variables. Thus, these variables are situated away from all other factors on the map. This is not a surprising result since it fits well our suggested model (these culture variables represent level one of Williamson's model as explained earlier and hence they are rather located "far" from the other levels) [51-54]. However, it is difficult to interpret the significance of this level vis-à-vis the other three levels of the model in more precise terms, one reason being that we only have available four of these culture variables (which are complex and composite in nature) and hence it is not certain how other similar variables might have behaved vis-à-vis the remaining factors [55]. Other variables which could also be considered as being part of "culture" are located in some other places on the map (e.g. Snacu).

A special group of variables is now worth mentioning. These variables are in particular social indicators (shown with the capital letter ' $S$ ' in front of each variable in Figure 3) that show social performance such as the 'Human Development Index' (Shdi) and the dependence ratio (Sdera). These are all but one (Squli, located near zero and to the west) situated on the Far East part of the map. They might be the consequences of all other variables on the map (although they might also be the cause for many of them). In a way we can say that all these social indicators represent a measure of governance in the sense that they depend on or are the cause of business and institutional governance (hence they may indicate a complex type of governance) [56-58]. However, we do not know to what extent and how institutional environment, governance, and economic performance all have an impact on these social indicators ${ }^{23}$. Can we also say that these "S" variables are not part of Williamson's model we refer to in this paper? We do not think so as we just proposed that they represent particular complex types of governance ${ }^{24}$.

${ }^{22}$ In our preliminary analysis the variable unemployment was also included, but its position in the map was an outlier, very far from all other variables. Although it is not now included in our maps, we may note that due to its extreme location unemployment might be a much more complex phenomenon that we think in economics. Thus, it could be considered as a social indicator (as also suggested by other scholars, e.g. see Chennareddy, 2010).

${ }^{23} \mathrm{We}$ want to emphasize again that it is out of the scope of this paper to find causa relationships between any groups of our variables.

${ }^{24}$ Since we have only 10 "S" variables and 13 "E" variables, some readers migh suggest that the $B$ and $G$ variables ( 30 and 32 respectively) are too many and may influence the results. Although we know that MDS cannot be influenced by the subtraction or addition of variables in the map (since relative distances remain overall the same), we also constructed some maps (not shown here) with an equal number of variables for each group. As expected the relative positions of the five groups remained the same.
Based on the analysis so far in this section we can now draw some conclusions on the meaning of the two dimensions ${ }^{25}$ or the four horizons of the map in Figure 3. Thus, we suggest that the further north ${ }^{26}$ we focus our attention the more institutional-oriented or sociopolitical becomes the content of the variables $[59,60]$. On the other hand the further south we go the more economic, labor, or cultureoriented in nature becomes the variable. Looking at the $\mathrm{x}$-axis (many more variables are aligned along this axis than along the $y$-axis), we observe that the further west we go the more institutional environment oriented the factors become; whereas the more we go on the east the more business governance oriented the factors become [61,62]. Close to the center of the map (close to zero) we might find variables which are more "balanced" in terms of the four horizons. Thus, the role of 'competition legislation' (Gcole) and 'worker motivation' (Bwomo) are two examples of such "balanced" variables. Note that this interpretation of the two axes can be adjusted according to the researcher's aims. Thus, the reader might be able to suggest a different interpretation from ours (though, we think it will not be too different from ours).

We now turn to partially verifying our MDS results by examining the actual dissimilarity matrix with all 88 pairs of distances (hence a matrix of $88 \times 88$ ). Given the low stress test and high $\mathrm{R}$ square of the MDS map (see note of Figure 3) we conclude that MDS closely represents the actual dissimilarity matrix. In other words, since the $\mathrm{R}$ square is very high (around 0.94 ) for the configuration of Figure 3, it will be a good illustration to show the actual dissimilarities matrix in relation to the 89 variables and their main 5 groups (G, B, C, E, and $S)$. For these five groups we calculated averages for each one of the 89 variables; an extract for 9 variables is shown in Table 3. Thus, for example, for the variable Bcrma, its average Euclidean distance with all other variables of group B is 1.819 according to the dissimilarity matrix (it is the minimum compared to all other 4 group averages); whereas its average correlation coefficient with all other variables of group B is 0.49 according to the similarity matrix (it is the maximum compared to all other 4 group averages); and so on for the other variables [63]. However, we cannot expect that all 89 variables follow similar patterns in relation to their own group and in relation to other groups. What we are more interested in are the relative distances between all variables as they are calculated through the algorithms of MDS and not absolute pair wise distances or correlations [64].

All these results so far as based on Williamson's model (as per see section 2) support our propositions. Thus, the location of the four categories (institutional, business governance, economic, and culture, plus social indicators) is clearly distinct on the map (with expected blurring effects on the boundary between groups, especially between $\mathrm{B}$ and $\mathrm{G}$ groups). To more clearly appreciate the distinct ${ }^{27}$ locations of the five groups on the map we also calculated the gravity centers for each one of the groups (indicated in Figure 3 with an arrow) ${ }^{28}$.Given this map in Figure 3, we propose now the circular schema as seen in Figure 4. Thus, we can start from any part of the circle and see the

${ }^{25}$ The MDS method does not provide a meaning of the $x$-axis and $y$-axis. It is up to the researcher to propose such meaning.

${ }^{26} \mathrm{n}$ some maps the reader might see the reversal of south-north or east-west; this is a normal outcome with MDS, in which cases the conclusions remain unchanged.

${ }^{27}$ Tests of mean equality were computed in terms of each axis separately and of the diagonal of the two axes between each group of variables. Thus, "distinct" gravity centers means that these centers are independent from each other according to these tests. In other words, as already mentioned, the $G$ variables are overall distinctly located on one side of the map and the $B$ variables on the other side of the map; and so on

${ }^{28} \mathrm{All}$ subsequent Figures showing maps include the gravity centers for the five groups of variables $B, C, E, G$, and $S$. 
Citation: Sanidas E (2017) Evidence of the Strong Nexus between Economic, Social, Business, and Political Indicators across the World. Int J Econ Manag Sci 6: 481. doi: 10.4172/2162-6359.1000481

Page 11 of 24

\begin{tabular}{|c|c|c|c|c|c|c|c|c|c|}
\hline Dissimilarity & Bcrma & Blare & Cindi & Cmasc & Einvp & Elapr & Gimla & Ginst & Sflad \\
\hline B & 1.819 & 1.698 & 2.429 & 2.740 & 2.980 & 2.275 & 2.311 & 1.808 & 2.108 \\
\hline C & 2.925 & 2.765 & 3.128 & 2.373 & 2.530 & 2.664 & 2.593 & 2.926 & 2.837 \\
\hline$E$ & 2.543 & 2.389 & 2.550 & 2.358 & 2.449 & 2.316 & 2.426 & 2.550 & 2.611 \\
\hline G & 2.001 & 1.771 & 2.181 & 2.779 & 2.876 & 2.008 & 2.277 & 1.763 & 2.336 \\
\hline $\mathrm{S}$ & 2.010 & 1.838 & 2.473 & 2.743 & 3.113 & 2.361 & 2.357 & 1.838 & 2.149 \\
\hline Similarity & Bcrma & Blare & Cindi & Cmasc & Einvp & Elapr & Gimla & Ginst & Sflad \\
\hline B & 0.490 & 0.530 & 0.363 & -0.149 & -0.111 & 0.458 & 0.213 & 0.505 & 0.283 \\
\hline $\mathrm{C}$ & -0.199 & -0.159 & -0.279 & 0.109 & -0.062 & -0.047 & -0.077 & -0.191 & -0.161 \\
\hline$E$ & 0.101 & 0.160 & 0.088 & -0.001 & -0.026 & 0.101 & 0.017 & 0.127 & -0.004 \\
\hline G & 0.456 & 0.570 & 0.479 & -0.194 & -0.126 & 0.559 & 0.251 & 0.627 & 0.207 \\
\hline $\mathrm{S}$ & 0.340 & 0.362 & 0.370 & -0.135 & -0.185 & 0.453 & 0.186 & 0.424 & 0.178 \\
\hline
\end{tabular}

Notes: The figures are averages of each variable in each of the five groups B, C, E, G, and S.

Table 3: Group distance or correlation averages (some examples).

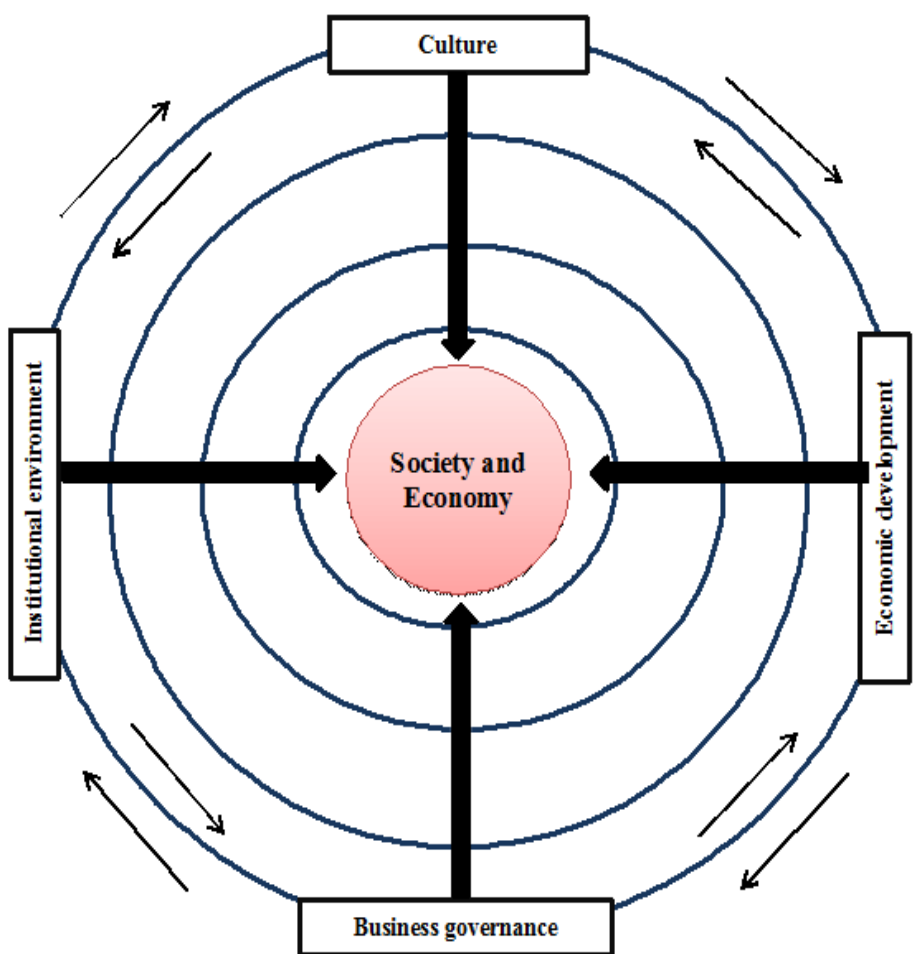

Figure 4: The four sets of socio-economic factors as per Williamson's model.

consequences along the circumference of this circle. For example, the institutional governance and business contract or business governance variables are next to each other and at a distance from economic variables. Note that any part of the circle is linked with any other part of the circle through direct diagonal links (thus indicating causation). Also, if we could add the dimension of time, then the relationships in Figure 4 would become like a DNA helix and thus they would become more dynamic in nature. The dark shaded center or core of this circle in Figure 4 can be considered as being the overall outcome in society.

To see all these groups in a more hierarchical way, we also applied the hierarchical clustering (HC) method [37] to the 89 (for 2011) variables. The resulting dendogram is shown in Figure 5 . We can see there that some distinct clusters are formed. Two distinct clusters are first noticed: first the cluster of culture variables plus three economic variables (A2 cluster). All the other variables are found in the other major cluster A1 which is nonetheless further split into 2 major sub- clusters: first the A11, the cluster of institutional environment (25 variables of the $G$ group, plus four variables of the $E$ group, plus two of the $\mathrm{B}$ group, and one of the $\mathrm{C}$ group, which are all closely located with the $25 \mathrm{G}$ variables); and second the $\mathrm{A} 12$ sub-cluster which comprises 28 out of 30 contract/business variables that represent business governance, plus all the $S$ variables, seven $G$ variables, and seven economic variables. Note that nine out of ten $S$ variables belong to the sub-cluster A1221 of A12. Also note that most of the economic variables $E$ belong to either first level cluster (A2) or third level sub-clusters (A112, A121). All G, B, and S variables belong to fourth level sub-clusters A1111, A1112, A1221, A1222, and $\mathrm{A} 1223$. Concerning the $\mathrm{C}$ factors, three out of four belong to the first level cluster A2. Overall the important conclusion to mention now is that the HC method agrees with the MDS method overall as expected. A more detailed analysis of these results is out of the scope of this paper.

\section{Clusters of economic development for countries}

So far we have examined the 89 (for 2011) socio-economic variables 


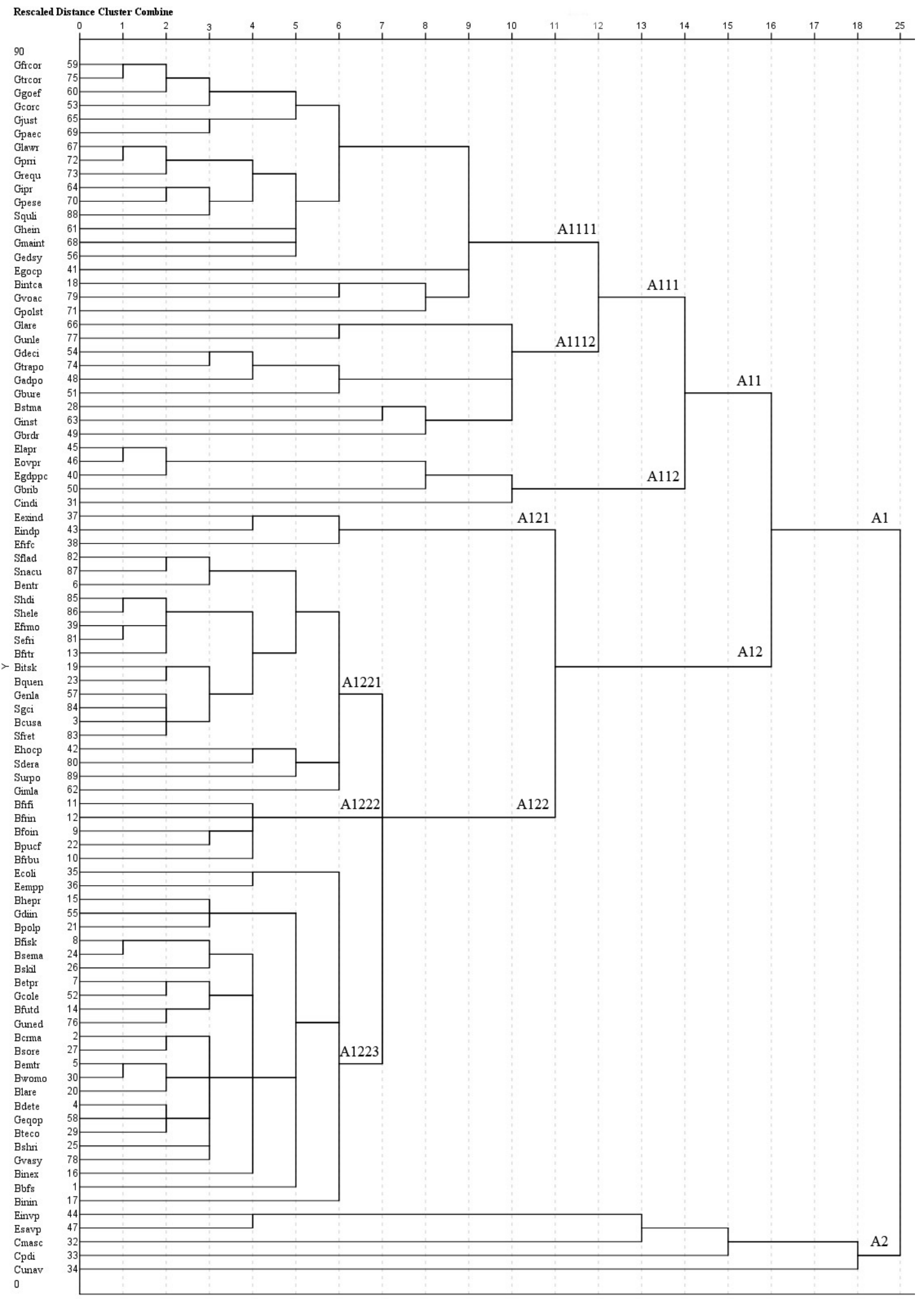

Notes: Squared Euclidean distance, 0-1 standardization, cluster method of between-groups.

Figure 5: 2011 map of 89 socio-economic indicators for 53 countries: dendrogram. 
and constructed a map that shows how all these variables are situated in relation to each other. This map is based on the underlying combination of 53 countries and their 89 corresponding variables. We can reverse this combination of countries and variables (by taking the transpose of relevant matrix) to have the corresponding image of countries on a map based on the 89 variables. In other words we can check the results of Figure 3 by their image (reflexion) on Figure 6; if the results of the map in Figure 3 make reasonable sense (as we just discussed it) then the results of the image map in Figure 6 (based on Euclidean distance, zero to one standardization again) should also make reasonable sense.

The map of these 53 countries as shown in Figure 6 is strikingly successful in truly (as expected) depicting the relative distances between our sample countries. A first overlook at this graph shows that on the west side of it we have the most advanced countries and on the east side we have the least advanced ones. Our aim would be now to separate all 53 nations on the map into three groups (which is a reasonable split) ${ }^{29}$. The preliminary criterion we can use is GDP per capita in PPP terms, which is also one of our economic variables (Egdppc). When we use this criterion, then we can have 17 to 18 countries in each one of the three groups. The first 18 countries in descending order include Norway (the highest) to Italy (the lowest); the full list can be seen on Table 4 in the appropriate column under "Egdppc". However, when we look at the map of Figure 6, we can see that if Italy should belong to the group 1 (the most advanced economies) then other countries to the west of Italy on the map (such as Spain and Israel) should also belong to this group; the same applies to Taiwan. On the other hand according to Figure 6, New Zealand is situated amongst the most advanced countries group and yet in terms of GDP in PPP terms per capita, it is further below Italy. Hence we decided to include only 17 countries in the first group from Norway down to Japan (as at 2011).

With a similar reasoning, Russia and Argentina are clearly on the east side of the map in Figure 6, and yet their GDP in PPP terms per capita is high enough to include these two countries in the second group of most advanced countries; symmetrically, Malaysia and Chile seem to belong to the second group according to the map in Figure 6 . Overall, then as seen in Table 4, the three groups are shown in the first column and they are separated by an oblique line ${ }^{30}$ in Figure 6 . We will keep this classification for the remaining of our study. In summary, in Table 4 we can see that our variable Egdppc is very successful in classifying countries into three groups according to the 89 socioeconomic variables. Thus, the Egdppc variable predicts correctly 16 out of 17 countries in group one, 14 out of 17 in group 2, and 17 out of 19 in group three. Obviously, at the border of the three groups we might have some countries that can belong to two groups at the same time. We will further justify the split into the three groups in the following paragraphs.

Thus, if we examine all 53 countries in the same way but only visà-vis the $\mathrm{B}$ variables, or $\mathrm{C}$, or $\mathrm{E}$, or $\mathrm{G}$, or $\mathrm{S}$ variables separately, we still obtain similar configurations as when we combine all variables $(\mathrm{B}$, C, E, G, and S) together, albeit with some significant differences; the corresponding results are shown in Figures 7A-7E. Although a detailed comparison is impossible here due to space limitations, nonetheless we can make some worthwhile comments. First, in terms of the B group of variables, Figure 7A shows that the above groups of most advanced 17 countries (called G1 group henceforth), middle income 17

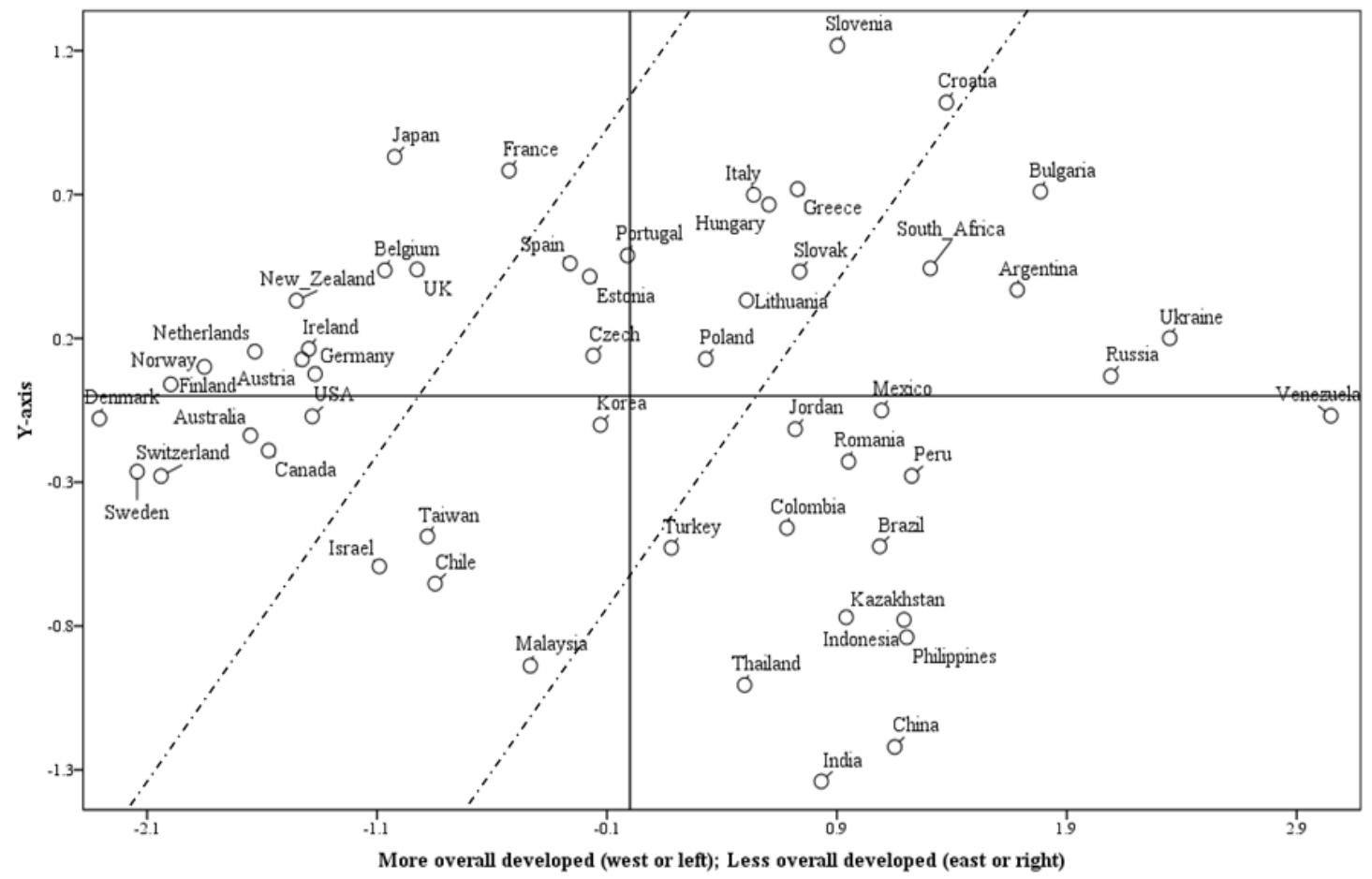

Notes: Euclidean distance, 0 to 1 standardization, stress $=0.09, \mathrm{R}^{2}=0.96$.

Figure 6: 2011 map in terms of 53 countries (as per 89 variables).

${ }^{29}$ Usually international organizations such as the World Bank split countries into three major groups.
${ }^{30} \mathrm{We}$ can rotate the map in any direction we want in order to interpret results, hence our rotation to the north-west and south-east axis as the oblique lines indicate on the graph. 
Citation: Sanidas E (2017) Evidence of the Strong Nexus between Economic, Social, Business, and Political Indicators across the World. Int J Econ Manag Sci 6: 481. doi: 10.4172/2162-6359.1000481

\begin{tabular}{|c|c|c|c|c|c|c|c|c|c|c|c|c|c|c|c|c|c|c|c|c|c|c|c|c|c|c|}
\hline lo & Country & G & Cou & Bintca & G & Cou & Egdppc & G & Cou & Eovpr & G & Cou & Gcorc & G & Cou & Gfrcor & G & Cou & Glawr & G & Cou & Shdi & G & Cou & Squli & \\
\hline & ustralia & 1 & we & 864.8 & 1 & Nor & 57017 & 1 & Nor & 111789 & 1 & Den & 2.42 & 1 & NZ & 94 & 1 & Fin & 1.96 & & Nor & 0.94 & 1 & Swi & 9.66 & 1 \\
\hline & ustria & & or & 862.99 & 1 & USA & 47572 & 1 & USA & 106098 & 1 & NZ & 2.33 & 1 & Den & 93 & & Swe & 1.95 & & Ausl & 0.93 & & Aus & 9.65 & 1 \\
\hline & Belgium & 1 & Den & 855.03 & 1 & Swi & 46739 & 1 & Ire & 100911 & 1 & Swe & 2.22 & 1 & Swe & 92 & & Den & 1.92 & & Net & 0.91 & 1 & Nor & 9.63 & 1 \\
\hline & anada & 1 & Net & 850.14 & 1 & Net & 42459 & 1 & Bel & 91896 & 1 & Fin & 2.19 & 1 & Swi & 90 & & NZ & 1.91 & & USA & 0.91 & 1 & Ausl & 9.4 & 1 \\
\hline & Denmark & 1 & Can & 848.62 & 1 & Aus & 41101 & 1 & Fra & 87402 & 1 & Net & 2.17 & & Fin & 89 & & Nor & 1.89 & & NZ & 0.91 & & Den & 9.21 & 1 \\
\hline & nland & 1 & Jap & 845.59 & 1 & Ire & 40666 & 1 & Net & 84698 & 1 & Nor & 2.17 & 1 & Net & 89 & & Net & 1.82 & & Can & 0.91 & 1 & Ger & 9.17 & 1 \\
\hline & France & 1 & Ausl & 843.43 & 1 & Den & 40462 & 1 & Ita & 84513 & 2 & Ausl & 2.16 & 1 & Ausl & 87 & 1 & Aus & 1.81 & & Ire & 0.91 & 1 & Bel & 9.17 & 1 \\
\hline & Germany & 1 & USA & 840.69 & 1 & Swe & 40267 & 1 & Aus & 83735 & 1 & Swi & 2.02 & 1 & Can & 87 & & Ausl & 1.78 & & Ger & 0.91 & 1 & Swe & 9.02 & 1 \\
\hline & Ireland & 1 & Fin & 840.3 & 1 & Can & 39725 & 1 & Den & 83258 & 1 & Can & 1.98 & 1 & Nor & 86 & 1 & Ire & 1.77 & & Swe & 0.9 & 1 & Net & 8.93 & 1 \\
\hline 10 & Japan & 1 & NZ & 838.57 & 1 & Ger & 38756 & 1 & Spa & 82598 & 2 & Ger & 1.69 & 1 & Ger & 80 & 1 & Swi & 1.76 & & Swi & 0.9 & 1 & Can & 8.9 & 1 \\
\hline 11 & Netherle & 1 & Kor & 827.97 & 2 & Ausl & 38430 & 1 & Swe & 82003 & 1 & Bel & 1.58 & 1 & Ire & 80 & & Can & 1.76 & & Jap & 0.9 & 1 & Fin & 8.78 & 1 \\
\hline 12 & ew Zealand & 1 & UK & 819.65 & 1 & Bel & 38189 & 1 & Fin & 81349 & 1 & Chil & 1.57 & 2 & Aus & 79 & 1 & Ger & 1.62 & & Kor & 0.9 & 2 & NZ & 8.71 & 1 \\
\hline 13 & Norway & 1 & Swi & 814.31 & 1 & Fin & 37337 & 1 & Tai & 80098 & 2 & Fra & 1.51 & 1 & Jap & 77 & 1 & UK & 1.59 & & Den & 0.89 & 1 & USA & 8.15 & 1 \\
\hline 14 & weden & 1 & Ger & 799.03 & 1 & Tai & 36933 & 2 & Can & 78897 & 1 & Jap & 1.5 & 1 & UK & 77 & & Fra & 1.5 & & Isr & 0.89 & 2 & Fra & 8 & 1 \\
\hline 15 & Switzer & 1 & Isr & 793.2 & 2 & UK & 35811 & 1 & Swi & 78429 & 1 & Ire & 1.5 & 1 & USA & 75 & 1 & Bel & 1.45 & 1 & Bel & 0.89 & 1 & Isr & 7.96 & 2 \\
\hline 16 & UK & 1 & Aus & 789.73 & 1 & Fra & 35639 & 1 & Ger & 76649 & 1 & Aus & 1.44 & 1 & Bel & 71 & 1 & Chil & 1.37 & 2 & Aus & 0.89 & 1 & Spa & 7.83 & 2 \\
\hline 17 & USA & 1 & Bel & 785.23 & 1 & Jap & 33402 & 1 & UK & 76628 & 1 & USA & 1.28 & 1 & Fra & 69 & & Jap & 1.27 & 1 & Fra & 0.88 & 1 & Ire & 7.61 & 1 \\
\hline 18 & Chile & 2 & Fra & 773.99 & 1 & Ita & 32168 & 2 & Ausl & 75997 & 1 & UK & 1.25 & 1 & Chil & 67 & 2 & Spa & 1.2 & 2 & Slov & 0.88 & 2 & Mal & 7.45 & 2 \\
\hline 19 & Croatia & 2 & Slov & 766.73 & 2 & Spa & 31689 & 2 & Isr & 75445 & 2 & Por & 1.09 & 2 & Est & 66 & 2 & Est & 1.18 & 2 & Fin & 0.88 & 1 & Cze & 7.42 & 2 \\
\hline 20 & Czech & 2 & Cze & 765.92 & 2 & Kor & 29610 & 2 & Gre & 73157 & 2 & Spa & 1.06 & 2 & Slov & 66 & 2 & Slov & 1.07 & & Tai & 0.88 & 2 & UK & 7.37 & 1 \\
\hline 21 & Estonia & 2 & Por & 764.44 & 2 & NZ & 29576 & 1 & Jap & 68318 & 1 & Slov & 0.93 & 2 & Isr & 61 & 2 & Tai & 1.04 & 2 & Spa & 0.88 & 2 & Jap & 7.3 & 1 \\
\hline 22 & Greece & 2 & Ita & 763.55 & 2 & Isr & 29143 & 2 & Kor & 60796 & 2 & Est & 0.91 & 2 & Spa & 61 & 2 & Por & 1.01 & & Ita & 0.87 & 2 & Tai & 7.16 & 2 \\
\hline 23 & Hungar & 2 & Est & 762.05 & 2 & Slov & 26856 & 2 & Slov & 58824 & 2 & Tai & 0.9 & 2 & Por & 58 & 2 & Cze & 1.01 & & Cze & 0.86 & 2 & Chil & 7.14 & 2 \\
\hline 24 & Israel & 2 & Tai & 753.71 & 2 & Gre & 26461 & 2 & $\mathrm{NZ}$ & 58477 & 1 & Isr & 0.68 & 2 & Tai & 56 & 2 & Kor & 1.01 & 2 & UK & 0.86 & 1 & Ita & 6.96 & 2 \\
\hline 25 & Italy & 2 & Ire & 753.66 & 1 & Cze & 24993 & 2 & Cro & 57910 & 2 & Pol & 0.51 & 2 & Kor & 55 & 2 & Isr & 0.98 & 2 & Gre & 0.86 & 2 & Kor & 6.32 & 2 \\
\hline 26 & Korea & 2 & Spa & 722.35 & 2 & Por & 24986 & 2 & Slok & 55588 & 2 & Kor & 0.45 & 2 & Hun & 51 & 2 & Hun & 0.77 & & Est & 0.83 & 2 & Tha & 5.95 & 3 \\
\hline 27 & Lithuania & 2 & Slok & 708.16 & 2 & Slok & 23996 & 2 & Hun & 55076 & 2 & Hun & 0.34 & 2 & Jor & 50 & 3 & Lit & 0.77 & 2 & Slok & 0.83 & 2 & Por & 5.94 & 2 \\
\hline 28 & Malaysia & 2 & Mal & 701.23 & 2 & Est & 22247 & 2 & Por & 54996 & 2 & Cze & 0.32 & 2 & Pol & 50 & 2 & Pol & 0.73 & 2 & Hun & 0.82 & 2 & Slov & 5.9 & 2 \\
\hline 99 & Poland & 2 & Hun & 667.95 & 2 & Hun & 20924 & 2 & Cze & 51766 & 2 & Slok & 0.29 & 2 & Cze & 49 & 2 & USA & 0.71 & 1 & Pol & 0.81 & 2 & Gre & 5.64 & 2 \\
\hline 30 & Portugal & 2 & Cro & 654.02 & 2 & Pol & 20731 & 2 & Tur & 51362 & 3 & Lit & 0.29 & 2 & Lit & 49 & 2 & Slok & 0.65 & 2 & Lit & 0.81 & 2 & Slok & 5.56 & 2 \\
\hline 31 & Slovak & 2 & Lit & 633.66 & 2 & Rus & 20588 & 3 & Est & 48940 & 2 & Bra & 0.17 & 3 & SA & 47 & 3 & Gre & 0.57 & 2 & Por & 0.81 & 2 & Tur & 5.24 & 3 \\
\hline 32 & Slovenia & 2 & Pol & 617.8 & 2 & Cro & 20124 & 2 & Pol & 48881 & 2 & Tur & 0.1 & 3 & Mal & 45 & 2 & Mal & 0.52 & & Chil & 0.8 & 2 & Ind & 5.07 & 3 \\
\hline 33 & Spain & 2 & Chil & 609.16 & 2 & Lit & 19984 & 2 & Lit & 46633 & 2 & SA & 0.03 & 3 & Slok & 45 & 2 & Ita & 0.41 & 2 & Arg & 0.8 & 3 & Arg & 4.91 & 3 \\
\hline 34 & Taiwan & 2 & Gre & 605.5 & 2 & Arg & 17071 & 3 & Arg & 45558 & 3 & Cro & 0.02 & 2 & Tur & 44 & 3 & Jor & 0.23 & 3 & Cro & 0.8 & 2 & Indo & 4.9 & 3 \\
\hline 35 & Argentin & 3 & Bul & 588.07 & 3 & Tur & 16738 & 3 & Rus & 41591 & 3 & Jor & 0.01 & 3 & Ita & 43 & 2 & Cro & 0.18 & 2 & Rom & 0.78 & 3 & SA & 4.9 & 3 \\
\hline 36 & Brazil & 3 & Rom & 499.21 & 3 & Chil & 16594 & 2 & Mal & 40395 & 2 & Mal & 0 & 2 & Cro & 41 & 2 & SA & 0.1 & 3 & Bul & 0.77 & 3 & Bra & 4.85 & 3 \\
\hline 37 & Bulgaria & 3 & Rus & 398.78 & 3 & Mex & 15503 & 3 & SA & 40345 & 3 & Ita & -0.01 & 2 & Bul & 38 & 3 & Tur & 0.08 & & Mex & 0.77 & 3 & Col & 4.81 & 3 \\
\hline 38 & China & 3 & Ukr & 377.49 & 3 & Mal & 15168 & 2 & Chil & 37713 & 2 & Gre & -0.15 & 2 & Gre & 38 & 2 & Rom & 0.04 & & Mal & 0.76 & 2 & Kaz & 4.79 & 3 \\
\hline 39 & Colombia & 3 & Jor & 373.27 & 3 & Rom & 14897 & 3 & Mex & 36149 & 3 & Bul & -0.17 & 3 & Rom & 38 & 3 & Bra & 0.01 & & Rus & 0.76 & 3 & Jor & 4.76 & 3 \\
\hline 40 & India & 3 & Ven & 356.95 & 3 & Bul & 14520 & 3 & Rom & 34559 & 3 & Rom & -0.2 & 3 & Bra & 37 & 3 & Ind & -0.08 & & Kaz & 0.74 & 3 & Est & 4.67 & 2 \\
\hline 41 & Indonesi & 3 & Tur & 354.29 & 3 & Kaz & 12806 & 3 & Bul & 31379 & 3 & Per & -0.2 & 3 & Col & 37 & 3 & Bul & -0.09 & & Ven & 0.74 & 3 & Pol & 4.63 & 2 \\
\hline 42 & Jordan & 3 & Bra & 352.37 & 3 & Ven & 12543 & 3 & Ven & 29661 & 3 & Col & -0.31 & 3 & Per & 37 & 3 & Tha & -0.24 & & Ukr & 0.73 & 3 & Cro & 4.62 & 2 \\
\hline 43 & Kazakhs & 3 & Per & 346.55 & 3 & Bra & 11630 & 3 & Kaz & 25722 & 3 & Mex & -0.36 & 3 & Chin & 36 & 3 & Col & -0.26 & & Per & 0.72 & 3 & Mex & 4.61 & 3 \\
\hline 44 & Mexico & 3 & Arg & 343.07 & 3 & SA & 10764 & 3 & Jor & 23312 & 3 & Tha & -0.37 & 3 & Ind & 34 & 3 & Chin & -0.46 & & $\mathrm{Bra}$ & 0.72 & 3 & Chin & 4.46 & 3 \\
\hline 45 & Peru & 3 & Tha & 329.49 & 3 & Col & 10048 & 3 & Col & 23153 & 3 & Arg & -0.39 & 3 & Tha & 34 & 3 & Mex & -0.48 & & Col & 0.71 & 3 & Per & 4.43 & 3 \\
\hline 46 & Philipp & 3 & Chin & 324.3 & 3 & Per & 9950 & 3 & Bra & 22914 & 3 & Ind & -0.56 & 3 & Mex & 33 & 3 & Phi & -0.51 & & Tur & 0.7 & 3 & Phi & 4.42 & 3 \\
\hline 47 & omania & 3 & Kaz & 314.85 & 3 & Tha & 8757 & 3 & Per & 19097 & 3 & Chin & -0.67 & 3 & Arg & 29 & 3 & Arg & -0.56 & & Jor & 0.7 & 3 & Lit & 4.12 & 2 \\
\hline 48 & Russia & 3 & Col & 289.86 & 3 & Chin & 8242 & 3 & Ukr & 15958 & 3 & Indo & -0.68 & 3 & Indo & 28 & 3 & Per & -0.6 & & Chin & 0.69 & 3 & Hun & 3.96 & 2 \\
\hline 49 & South Afri & 3 & Mex & 285.68 & 3 & Ukr & 7096 & 3 & Tha & 15387 & 3 & Phi & -0.78 & 3 & Kaz & 27 & 3 & Kaz & -0.63 & & Tha & 0.68 & 3 & Rus & 2.86 & 3 \\
\hline 50 & Thailand & 3 & SA & 203.41 & 3 & Jor & 5730 & 3 & Chin & 14532 & 3 & Ukr & -0.98 & 3 & Phi & 24 & 3 & Indo & -0.65 & & Phi & 0.64 & 3 & Rom & 2.79 & 3 \\
\hline 51 & Turkey & 3 & Indo & 200.33 & 3 & Indo & 4661 & 3 & Phi & 10323 & 3 & Kaz & -1.01 & 3 & Rus & 22 & 3 & Rus & -0.78 & 3 & SA & 0.62 & 3 & Bul & 2.08 & 3 \\
\hline 52 & raine & 3 & Phi & 165.86 & 3 & Phi & 4096 & 3 & Indo & 10071 & 3 & Rus & -1.09 & 3 & Ukr & 22 & 3 & Ukr & -0.86 & & Indo & 0.62 & 3 & Ven & 1.93 & 3 \\
\hline 53 & Venezuela & 3 & Ind & 133.13 & 3 & Ind & 3705 & 3 & Ind & 9759 & 3 & Ven & -1.22 & 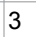 & Ven & 19 & 3 & Ven & -1.62 & & Ind & 0.55 & 3 & Ukr & 1.78 & \\
\hline
\end{tabular}

Notes: The first column indicated with "G" is the classification of countries chosen according to Figure 6 . For the other columns indicated "G", we can see the predicted capacity of each one of the indicators. Thus the "Human Development Index (Shdi) predicts the "Advanced group" of 17 countries as $15 / 17$ ? correct etc.

Table 4: Classification of countries based on selected variables (2011). 
Citation: Sanidas E (2017) Evidence of the Strong Nexus between Economic, Social, Business, and Political Indicators across the World. Int J Econ Manag Sci 6: 481. doi: 10.4172/2162-6359.1000481

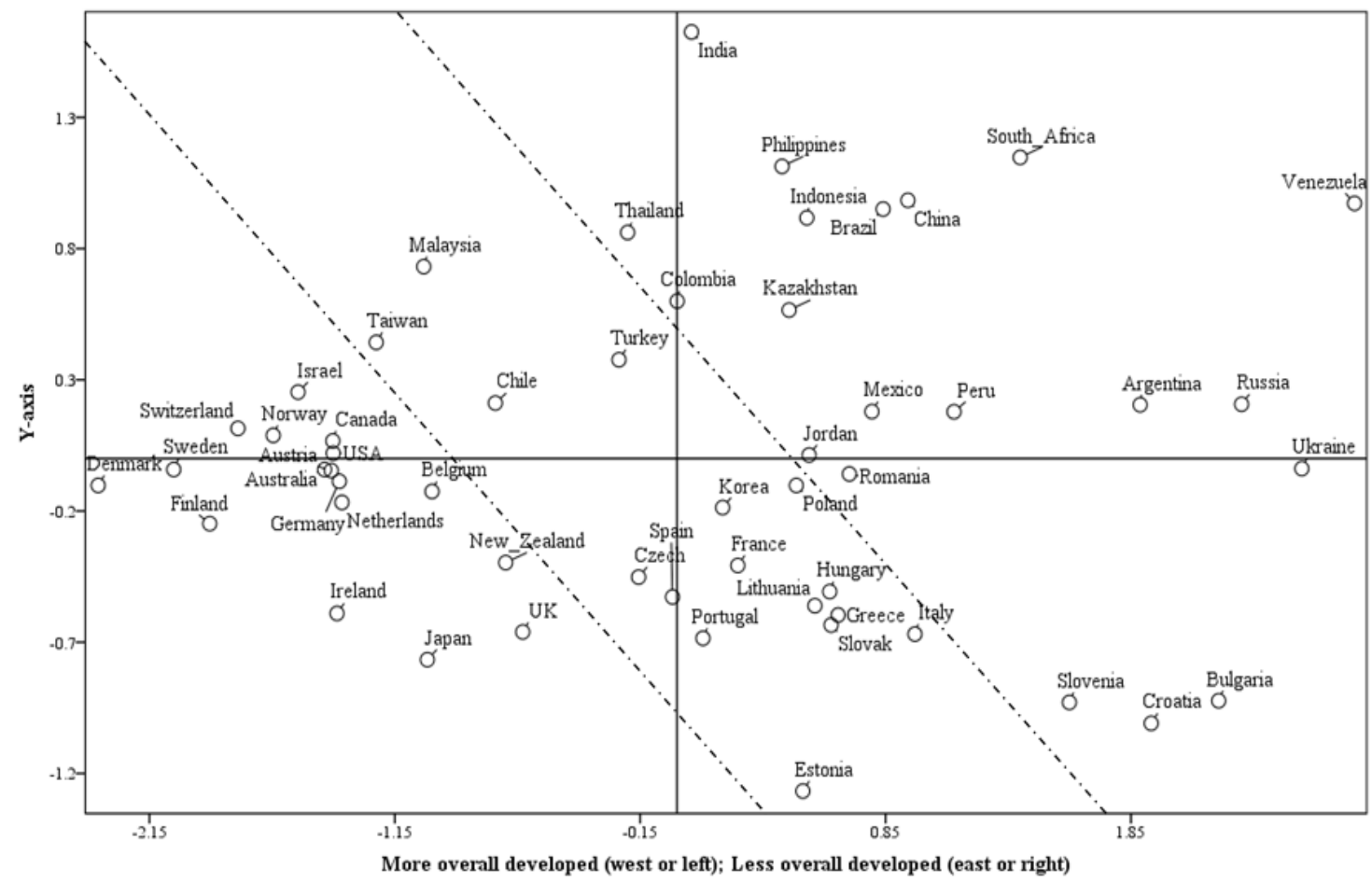

Notes: Euclidean distance, 0 to 1 standardization, stress $=0.11, \mathrm{R}^{2}=0.95$.

Figure 7A: 2011 map of 30 business governance indicators (B) for 53 countries.

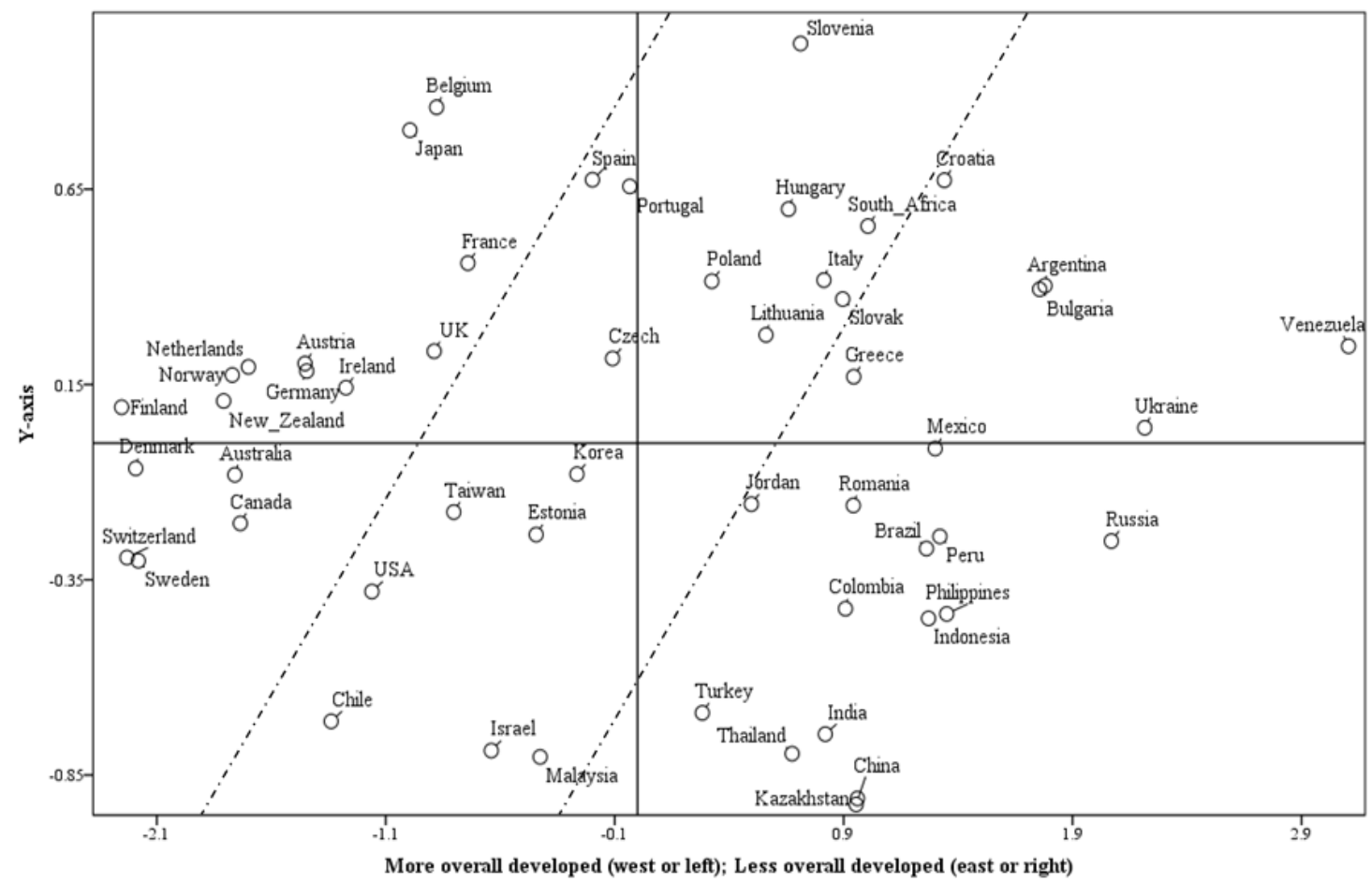

Notes: Euclidean distance, 0 to 1 standardization, stress $=0.07, \mathrm{R}^{2}=0.98$.

Figure 7B: 2011 map of 32 governance [institutional environment] indicators $(G)$ for 53 countries.

countries (called G2), and least advanced 19 countries (called G3) are approximately the same. The exceptions are few but worth commenting on: France is clearly situated in the G2 group, whereas Israel is located in G1; on the other hand Turkey is rather part of the G2, and Slovenia 
and Croatia in the G3 group. We can then recommend that France ought to improve its B variables, and so on. Second, in terms of the $G$ set of variables (Figure 7B), there are two noticeable exceptions to the countries belonging to each group G1, G2, and G3: the USA are away from most other nations in G1 and most probably belong to G2; Greece is rather clearly in G3 instead in G2.

Third, in terms of the E set of variables (Figure 7C), there are six noticeable exceptions: Italy is rather in G1 group instead of G2, Canada and New Zealand are rather in G2 instead of G1, Russia is rather in G2 instead in G3, whereas Chile and Malaysia are rather in G3 instead of G2. Fourth, in terms of the S set of variables (Figure 7D), there is one noticeable exception, that of Ireland which most probably belongs to G2 group instead of the G1 group. Fifth, in terms of the C set of variables (Figure 7E), there are several noticeable exceptions: France is rather part of G2 instead of G1; Korea and Japan are part of G3 instead of G2 and G1 respectively; Portugal, Greece, Korea, Slovenia, and Slovak Republic are rather situated in G3 instead of G1; Argentina, India, and Brazil are located in G2 instead of G3. This relatively high number of aberrant cases of nations belonging to different groups is not surprising as cultural traits change very slowly despite other changes in the society. Overall, the above results are very important because they show once more that all socio-economic variables grow in tandem through time albeit some exceptions), thus leading to the same overall development; in particular they show that the five sets of variables (B, C, E, G, and S) grow as relatively autonomous groups and yet interdependent at the same time (although the $\mathrm{C}$ set is rather more aberrant in its behavior). In addition, there are a few countries which are not consistently part of the same group G1, G2, or G3. variables. Unfortunately, we do not have enough data, in other words the ratio between the number of countries and the number of variables should be about 5 to 1 in order to get statistically significant results. Hence, alternatively we will check the above results as follows. First, we carried out HC based on the 89 variables in order to cluster countries; we tried several methods such as the Ward or centroid clustering, and several distance measures such as Minkowski's or block. They all show similar patterns of country clusters (with some minor differences). In Figure 8 we show the results using average linkages (within groups), zero to one standardization, and Euclidean distance. The dendogram in Figure 8 distinguishes several groups. The main group A1 comprises two subgroups, the A11 of all advanced nations as seen in G1 of Figure 6 except France plus two nations of the G2 of Figure 6 (Israel and Chile); and the A12 subgroup which comprises three more countries of G2 of Figure 6 (Malaysia, Taiwan, and Korea). Hence according to the $\mathrm{HC}$ tool, the A1 group in Figure 8 as just described encompasses what we may call the super group of advanced nations (21).

In the other major group A2 we have three subgroups: the A222 and A21 subgroups include all the least advanced nations of G3 of Figure 6 plus Croatia ( 20 countries). Finally the group A221 comprises most of G2 nations of Figure 6 plus France ${ }^{31}$ (12 countries). In summary, the MDS and HC multivariate models (as shown in Figures 6 and 8 respectively) provide similar results in terms of $\mathrm{G} 1$ and $\mathrm{G} 3$ groups, but not in terms of the G2 group as five nations of the G2 group of the MDS model now belong to the most advanced nations group A1. This is not a surprising result as countries in the middle of socio-economic development can also be considered as part of the neighboring groups. Are the clusters of $\mathrm{HC}$ as shown in Figure 8 a better representation

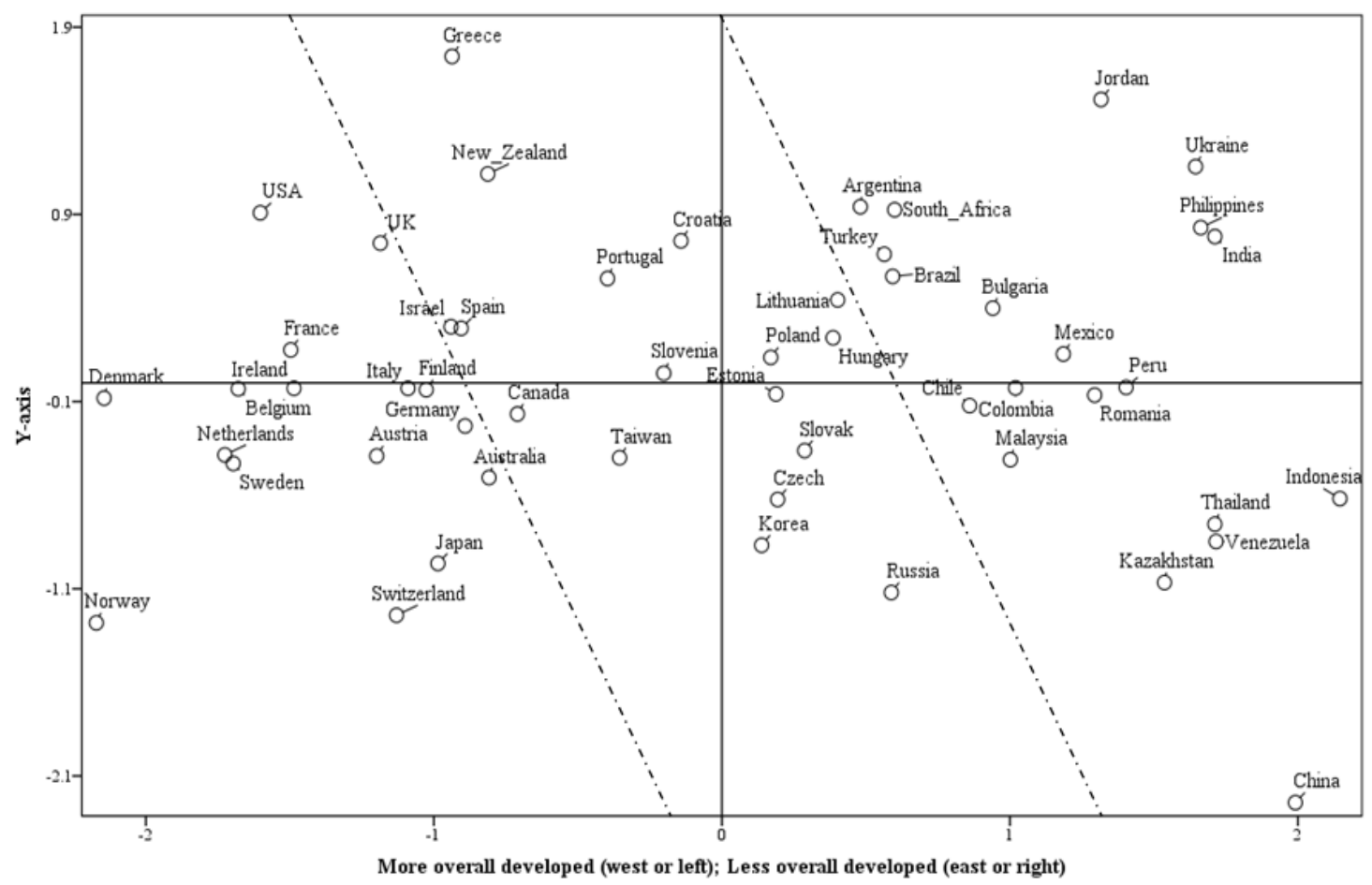

Notes: Euclidean distance, 0 to 1 standardization, stress $=0.11, R^{2}=0.94$.

Figure 7C: 2011 map of 13 economic indicators (E) for 53 countries.

To further check our results in Figure 6, we could have applied discriminant analysis or factor analysis to the 53 countries and 89
${ }^{31}$ All tried alternatives of $\mathrm{HC}$ consistently do not include France into the advanced group of nations; so perhaps we should eliminate this nation from the G1 group. 
Citation: Sanidas E (2017) Evidence of the Strong Nexus between Economic, Social, Business, and Political Indicators across the World. Int J Econ Manag Sci 6: 481. doi: 10.4172/2162-6359.1000481

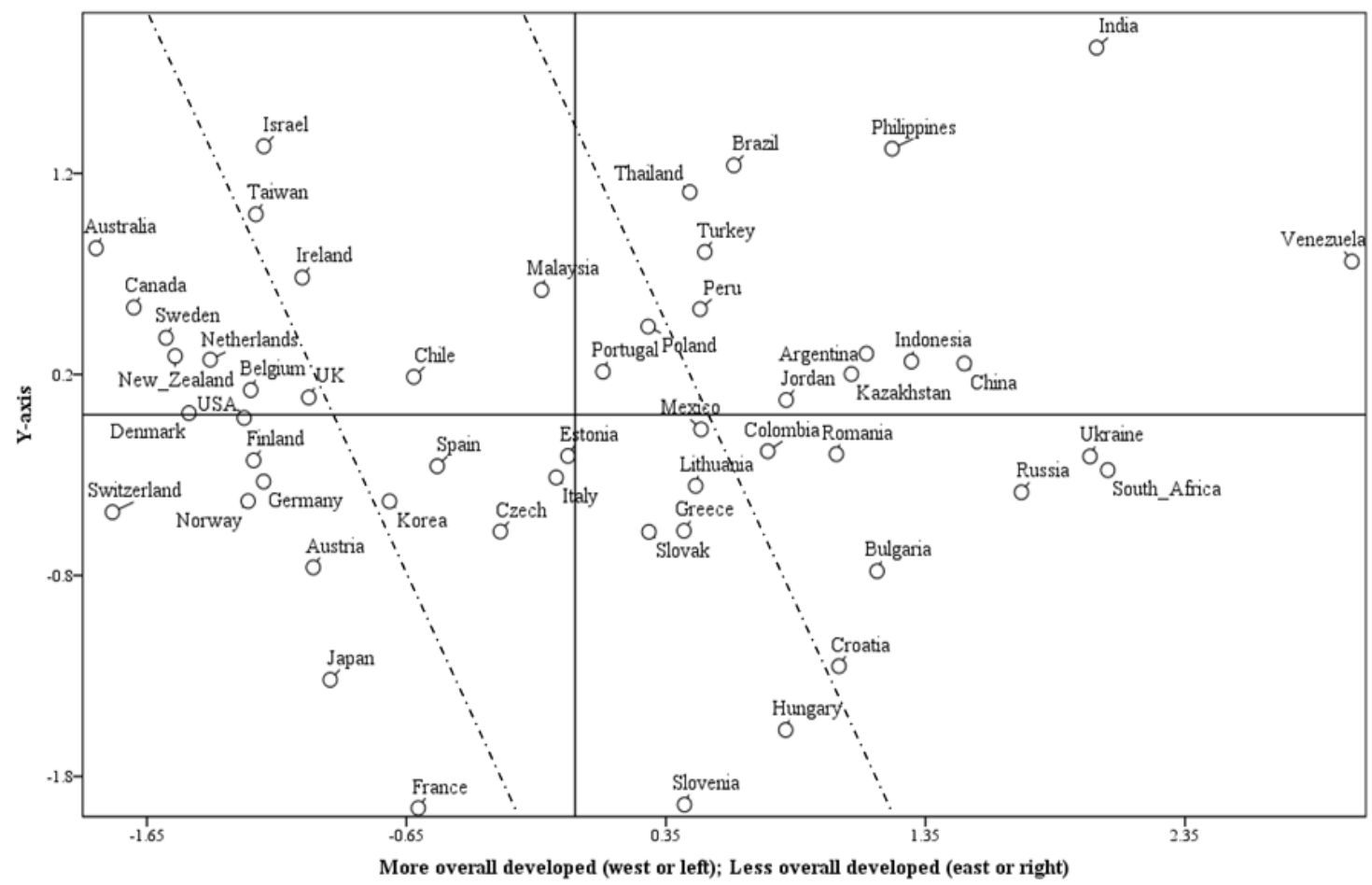

Notes: Euclidean distance, 0 to 1 standardization, stress $=0.14, \mathrm{R}^{2}=0.91$.

Figure 7D: 2011 map of 10 social indicators (S) for 53 countries.

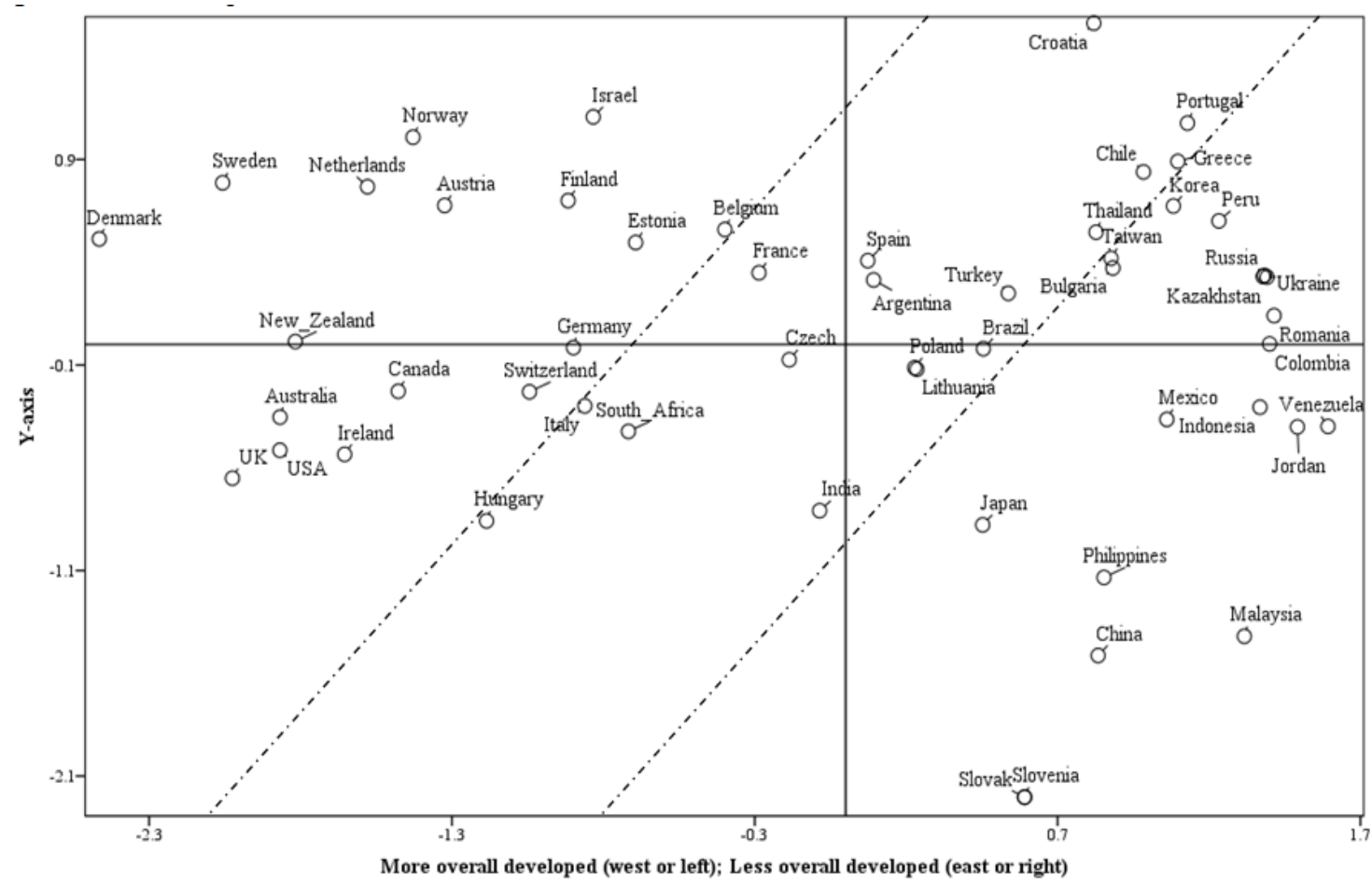

Notes: Euclidean distance, 0 to 1 standardization, stress $=0.16, R^{2}=0.88$.

Figure 7E: 2011 map of 4 culture indicators (C) for 53 countries. 


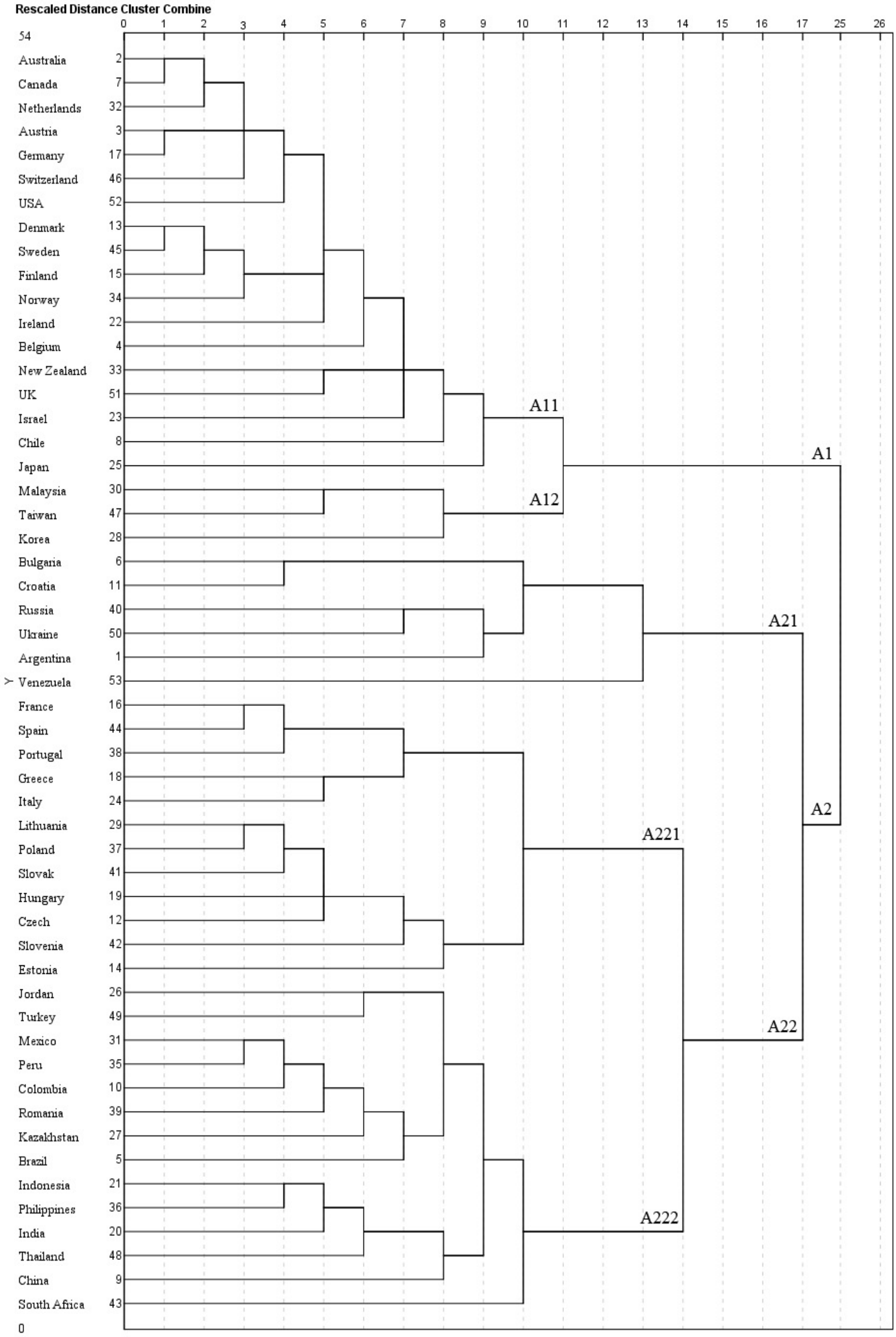

Notes: Squared Euclidean distance, 0-1 standardization, cluster method of within-groups.

Figure 8: 2011 map of 53 countries based on 89 socio-economic indicators. 
Citation: Sanidas E (2017) Evidence of the Strong Nexus between Economic, Social, Business, and Political Indicators across the World. Int J Econ Manag Sci 6: 481. doi: 10.4172/2162-6359.1000481

of classification of socio-economic development than the three groups G1, G2, and G3 of the MDS as shown in Figure 6? There is no definite and clear answer to this question; however for the remaining of our study we will adopt the classification into G1, G2, and G3 groups as it is further below justified.
Second, if we accept that the split between the three groups G1, G2, and G3 is correct, then we can construct the maps for each one of these three groups in terms of the 89 variables. The results are shown in Figures 9A-9C. The respective maps are in general similar to that of Figure 3. However, there is a noticeable tendency: as we pass from

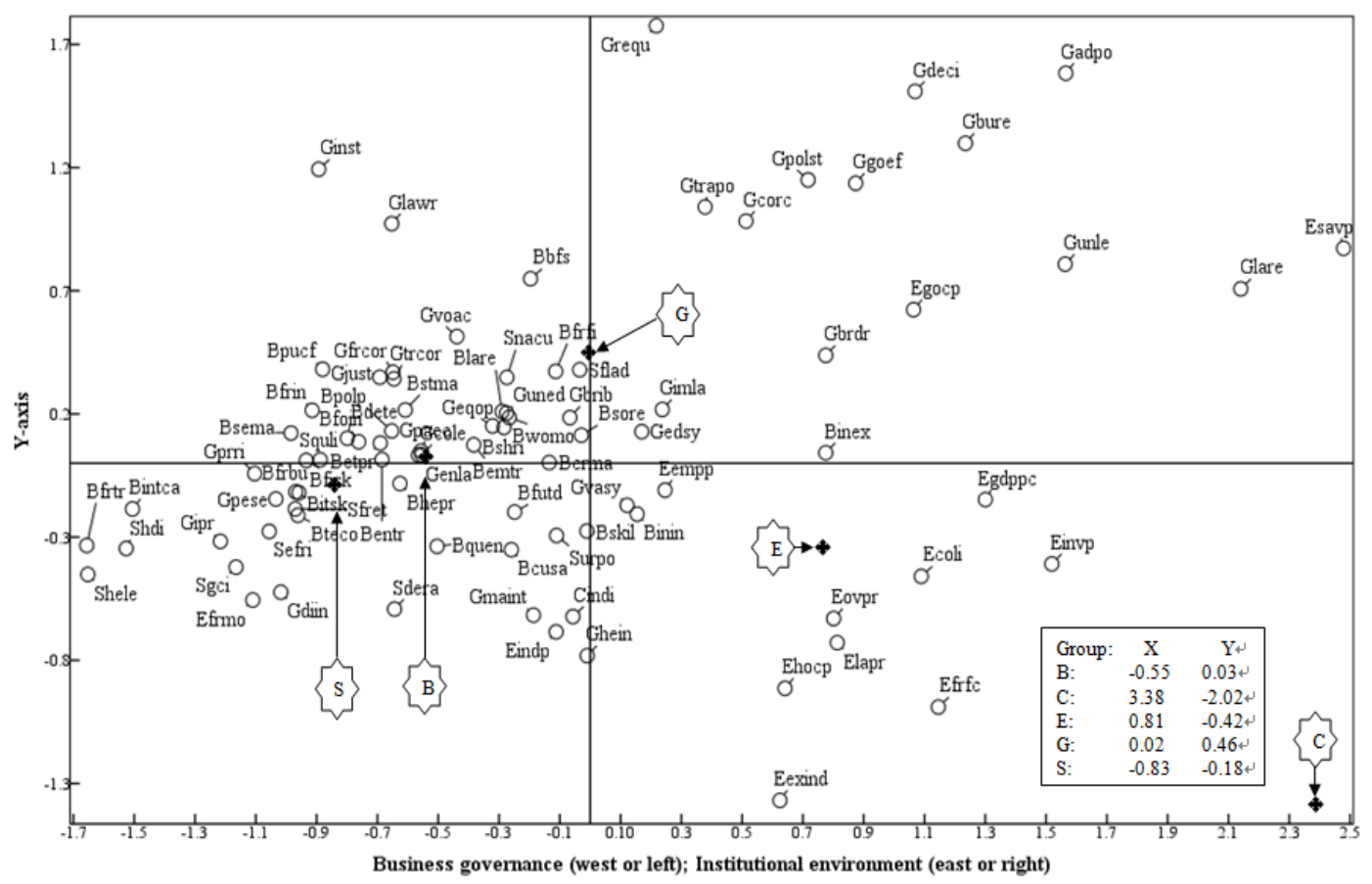

Notes: Euclidean distance, 0 to 1 standardization, stress $=0.16, R^{2}=0.94$.

Figure 9A: 2011 map of 89 socio-economic indicators for Group 1 of 17 countries.

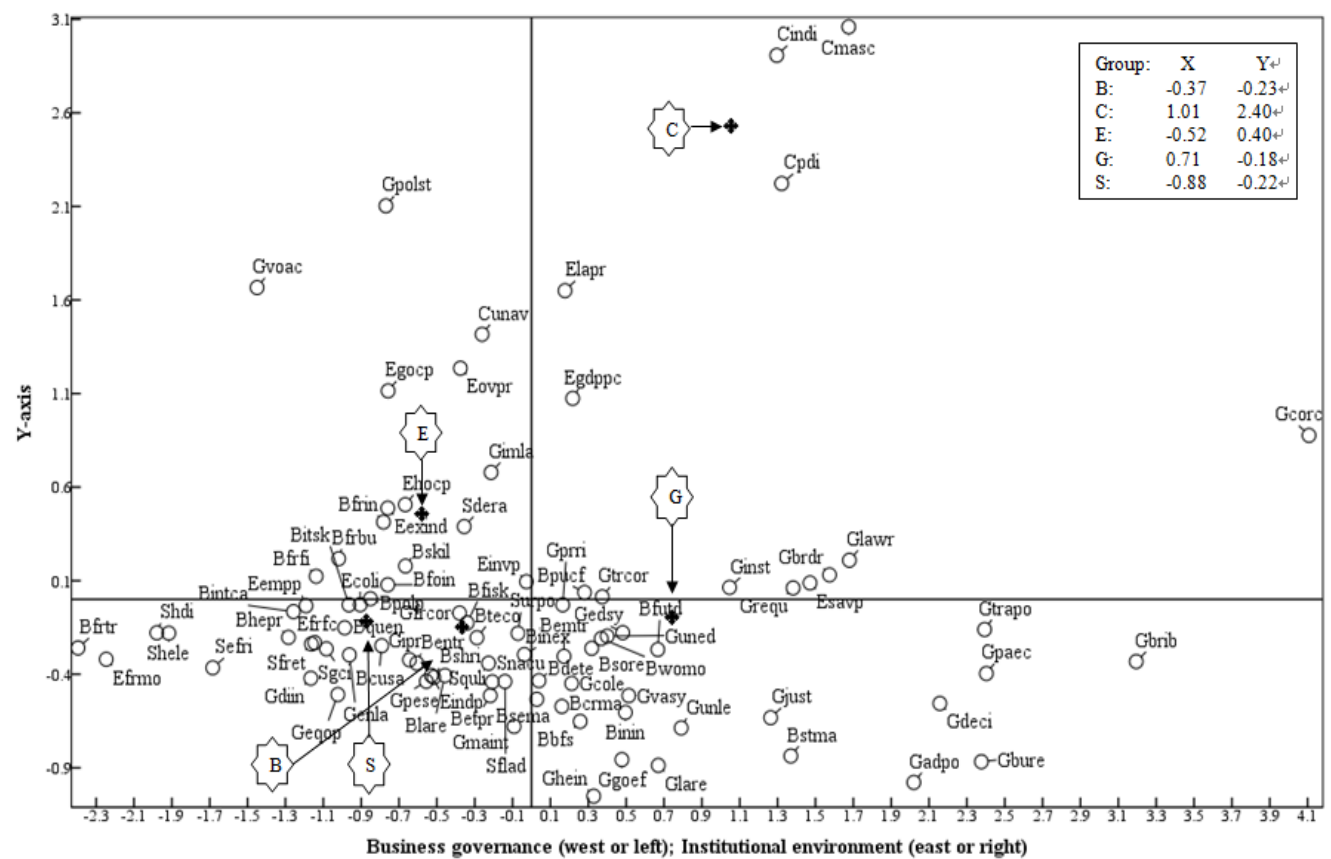

Notes: Euclidean distance, 0 to 1 standardization, stress $=0.16, R^{2}=0.92$.

Figure 9B: 2011 map of 89 socio-economic indicators for Group 2 of 17 countries. 


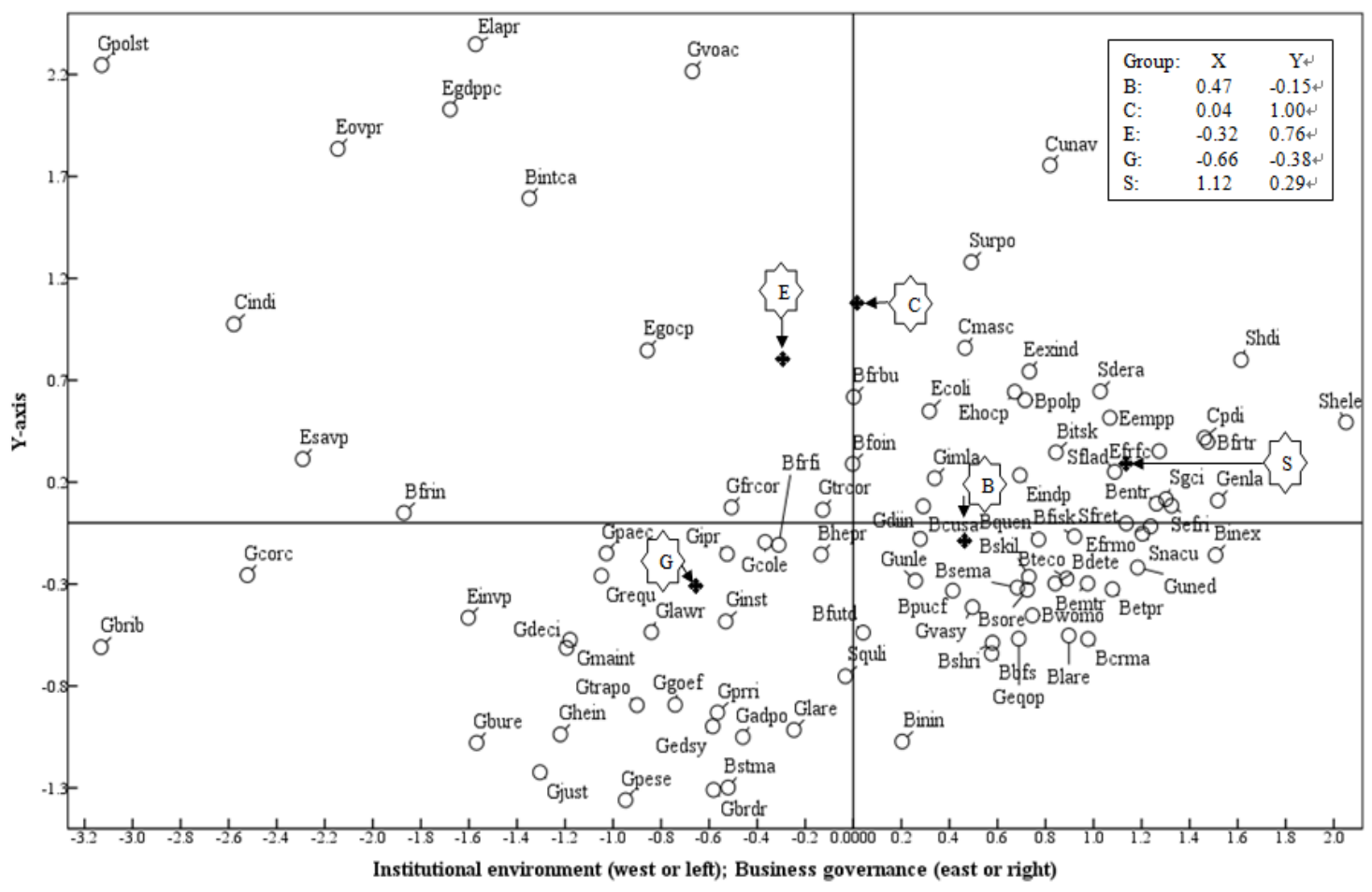

Notes: Euclidean distance, 0 to 1 standardization, stress $=0.17, \mathrm{R}^{2}=0.89$.

Figure 9C: 2011 map of 89 socio-economic indicators for Group 3 of 19 countries.

group 3 to group 2 and to group 1 the distances between the G, B, and $\mathrm{S}$ sets diminish in terms of their gravity centers. Thus, especially the $B$ and $G$ variables are closer in G1 than in G3 and more G variables are situated in B's territory (and vice-versa). This can be explained by arguing that as countries become more advanced overall in terms of socio-economic development, the institutional environment is more efficient in assisting business in conducting their economic activities. Nonetheless, separate G, C, E, B, and S groups are still spontaneously formed on the map regardless of the stage of development the countries are at.

Third, we took 8 variables (about one to three from each of the four categories of variables, thus excluding group C) and compared their value rankings with the three development groups of countries as suggested above based on Figure 6 and Table 4. Thus if our classification is correct, the top 17 rankings of a particular variable should belong to the top 17 countries as shown in Figure 6, and so on for the other two categories (17 and 19 countries respectively). All of these chosen variables fit the proposed classification (17-17-19) relatively well. Amongst these eight indicators ${ }^{32}$ (e.g. Gcorc, Bintca, and Glawr), the standard economic variable Egdppc (GDP per capita in PPP terms), which is usually used by economists to indicate overall economic development, confirms this role in our analysis; in other words it is not wrong to use GDP per capita (in PPP terms) in order to classify economic development as it accurately represents our 89 socioeconomic variables.

In addition, other non-economic variables similar to those in Table 4 are very good indicators of development classification. We extended

${ }^{32}$ Other researchers have attempted to use another indicator (other than GDP per capita) as a dependent variable for explaining development (see for example Knowles and Owen, 2010, who used "life expectancy"). this analysis to all 89 variables (not shown here for space limitations). As expected not all of them represent the proposed classification adequately. Many other variables of the G group are also very good single indicators: Gbrib (IMD), Gdiin (IMD), Ggoef (WGI), Gipr (IMD), Gjust (IMD), Gpese (IMD), Gpolst (WGI), Gprri (Heritage), Grequ (WGI), Gtrcor (Transperancy International), and Gvoac (WGI). Although variables of the B group are not as good single classification indicators as those of the $\mathrm{G}$ group, the following ones stand out: Bdete (IMD), Betpr (IMD), Bfrbu (Heritage), Bhepr (IMD), Bitsk (IMD), Bshri (IMD), and Bteco (IMD). In terms of the $S$ group, the following three are also worth mentioning: Sefri (Cato), Sgci (WEF), and Shele (IMD). In terms of Hofstede's C variables, only Cindi could be used as a relatively good single indicator of socio-economic development classification. Finally, most economic variables are not good single development indicators, the exceptions being Elapr, Egocp, and Efrmo (Heritage). The poor performance of many economic variables could be due to the non-linear relationship between them and economic development; for example, investment as a percentage of GDP (our variable Einvp) is high when countries take off economically and then it slows down as countries achieve high standard livings.

\section{Some comparative statics}

We will finish this section by comparing our results for 2011 (as described so far) with our analysis for $1995^{33}$. This comparison through the course of 15 years will provide us with some idea as to whether relative positions of countries and variables in the process of economic development remain stable through time. Not all results will be shown here as this would add much space to this paper. Only the main

${ }^{33}$ We also did some comparisons with the years 2000 and 2008 (not shown here) but the conclusions remain the same as for the comparison between 1995 and 2011. 
Citation: Sanidas E (2017) Evidence of the Strong Nexus between Economic, Social, Business, and Political Indicators across the World. Int J Econ Manag Sci 6: 481. doi: 10.4172/2162-6359.1000481

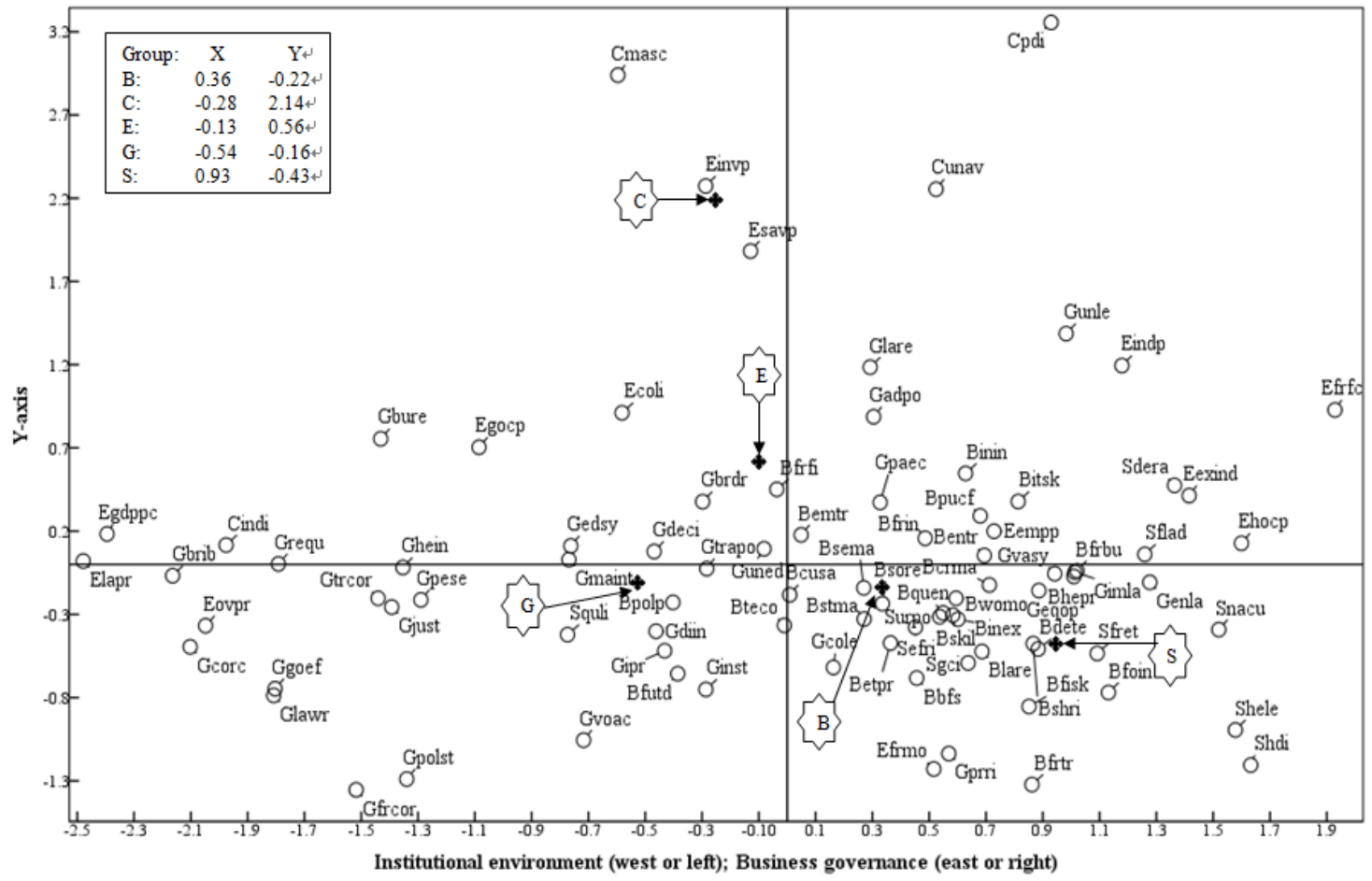

Notes: Euclidean distance, 0 to 1 standardization, stress $=0.14, R^{2}=0.93$.

Figure 10A: 1995 map of 89 social indicators for 43 countries.

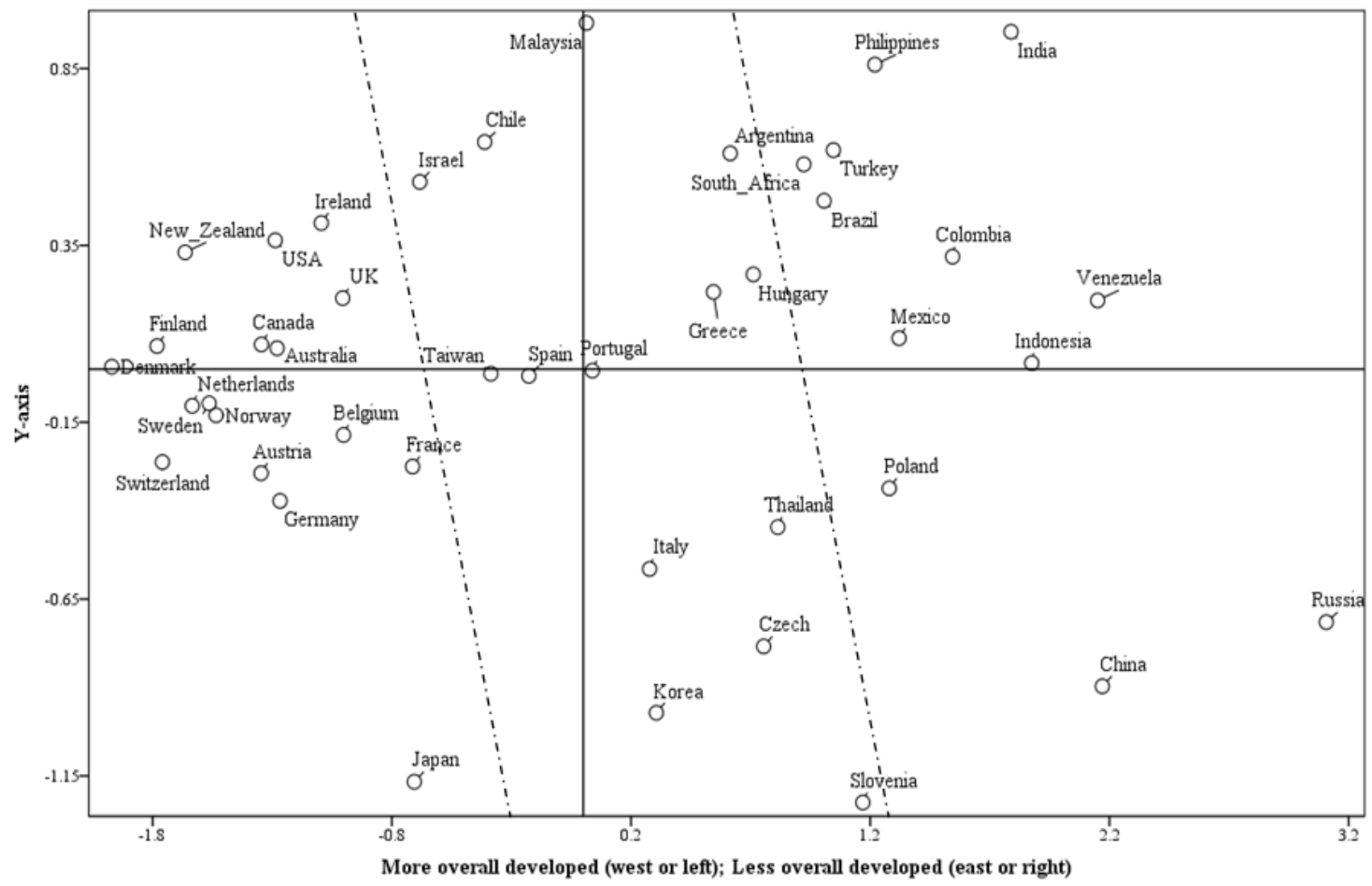

Notes: Euclidean distance, 0 to 1 standardization, stress $=0.12, \mathrm{R}^{2}=0.95$.

Figure 10B: 1995 map of 43 countries (89 socio-economic indicators). 


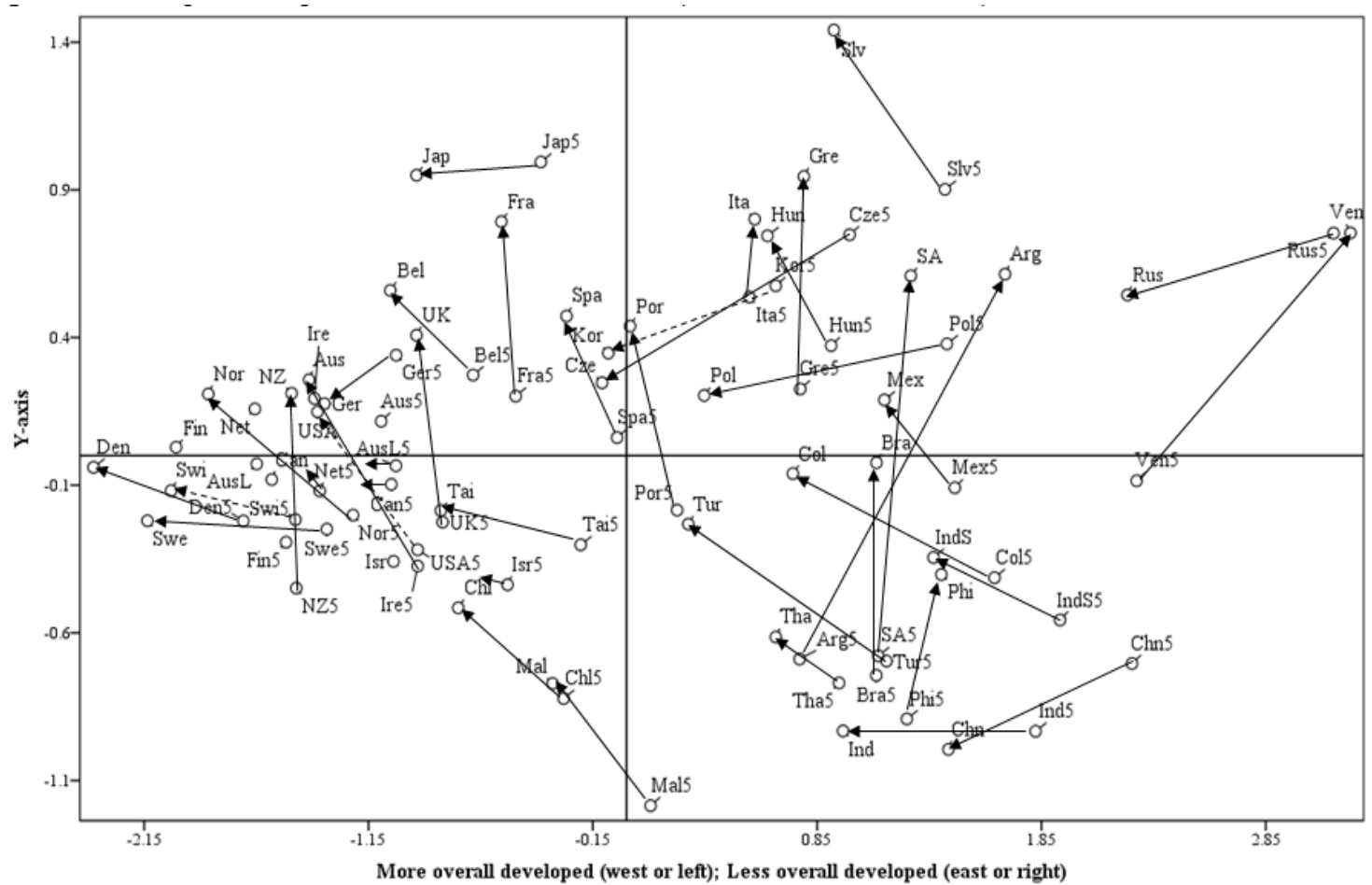

Notes: Euclidean distance, 0 to 1 standardization, stress=0.13, $R^{2}=0.93$, Arg stands for 2011, Arg5 for 1995.

Figure 11: Comparison map of 43 countries for 1995 and 2011 (89 socio-economic indicators).

differences will be briefly described. Also note that the definitions of variables for IMD, especially those that are based on surveys usually change from time to time and hence comparisons should take that into account when concluding (however, the IMD data used here are extracted from a supposedly consistent recently published time series data basis). Furthermore, for the year 1995 we replaced some missing data from adjacent years (e.g. 1996 or 1997) for some variables; in addition for 1995 we only have data for 43 countries. Figure 10A shows the 89-variable map for 1995 and Figure 10B shows the 43-country map for 1995. In terms of the 89 (or 90 for 2000 because of an extra variable for technology, Bteca, but graph not shown here) indicators, maps 10A and 3 do not show any significant differences, thus confirming the relative separate position of the five groups of variables from 1995 to 2011.

In terms of the 43 countries comparison between 1995 and 2011 (53 countries for this year), we can still see for 1995 the same three groups G1, G2, and G3 as suggested for 2011. This implies that it is not easy at all in the medium term of 15 years for a country to catch up so much as to belong to a more advanced group. To check this last statement we included both maps for the years 1995 and 2011 into one map (Figure 11); hence the movements of each country between 1995 and 2011 can easily be seen in this Figure 11. Thus we can observe that almost all countries moved forward from east to west or to southwest or to north-west; the exceptions being Venezuela and Argentina which moved back to north-east, and Philippines, South Africa, Brazil, Greece, New Zealand, and Italy which moved almost straight to the north (indicating influence of a particular set of variables). The exact changes in distances (calculated by applying Pythagoras' theorem) for each country are shown in Appendix 1. The largest socio-economic catch up took place for the countries (in descending order): Czech Republic, Poland, Turkey, Colombia, China, India, Russia, Norway,
Sweden, Korea, and so on. The most outstanding conclusion of the above comparison is that changes seem to be very slow; thus it is not easy to change camp easily (say from group three to two or from group two to one), although small differences within each group may be possible.

\section{Conclusions}

All our three propositions as set out in section 1 are verified in our study. First, one of the most important findings of this paper is the validation of Proposition 2. Our quantitative analysis of MDS and HC shows that 89 socio-economic variables that encompass the whole spectrum of institutions, governance, contracts, education, technology, culture, and economic performance represent our expectations we have about the 53 countries' overall performance. Thus, the categorization of overall economic and societal development into three main groups (advanced, semi-advanced, and less advanced) is examined and cross-checked in several ways. A corollary conclusion is that several individual variables such as government efficiency, GDP per capita, and others can also represent this categorization. For example, these 89 socio-economic variables tell us that Argentina belongs to the third group of less developed countries in the same way as Argentina's GDP per capita tells us so.

Second, another important conclusion and finding is that Williamson's model of economic institutions and their interactions with societal development is overall correct (Proposition 1). This is so because his four levels of social analysis are overall confirmed with our quantitative analysis. We have effectively discerned (through using the technique of MDS and HC) four distinct groups of variables that closely correspond to the four levels of analysis in Williamson's model (we introduced a fifth group in this model, namely social indicators). 
These four groups are located in a relatively distinct ${ }^{34}$ part of the map as shown in various relevant Figures, thus indicating that in general variables within each group are more correlated or in closer distance than variables between each group ${ }^{35}$. Also, there exists a loose hierarchy in terms of subgroups of variables as shown in Figure 5 based on HC.

Third, another important conclusion is that economic development goes in tandem with social, institutional, contractual, and generally societal development (Proposition 3). This was shown with the use of multivariate methods such as MDS and HS which can tell us that the grouping of 89 (for 2011, 90 for 2000, and 89 for 1995) socio economic variables into $4-5$ clusters is valid given that the suggested grouping of countries is valid as well (as per economic development). In other words, the whole society develops in several directions at the same time (in tandem): appropriate institutions, business governance and attitudes, education, infrastructure, and culture are all conjointly moving in time to reach compatible levels of development. However, not all variables within each major group grow in the same way, or the same direction.

The validation of Williamson's model is also checked in a more dynamic way. The map of $89-90$ socio-economic variables of the five categories (institutional environment, business governance, economic performance, and culture, plus composite social indicators) and the corresponding map of economic development of our 43 to 53 countries sample (in 1995 we have 43 countries and 89 variables) do not change easily though time. Thus, through comparisons between 2011 and 1995 we get virtually the same results. Only small differences may be observed mainly within each one of the three groups of socio-economic development. All this shows that only in the very long term (probably more than 50 years) we might expect a substantial change in the overall socioeconomic position of countries. This is mainly due to the very slow changes of institutional, governance and culture variables through time (as Williamson's model predicts). As hinted at in Introduction, another way of expressing this parallel development of all these 89 indicators is that a market economy can function only in an appropriate market society (appropriate in terms of institutions, governance, and so on).

However, there are some limitations in this study: first, in our sample of countries included in the analysis, due to data limitations, several countries were not included. Second, regressions and other types of standard analysis are not performed, thus making our results perhaps less credible in the eyes of standard economics scholars who would like to think in terms of regressions. The two main reasons why regressions were not included here are first space limitations, and second in this preliminary study we wanted to show the overall mapping of all variables considered together as explained earlier. Third, some readers might object to us having used so many surveybased variables (like those produced by IMD), but when dealing with socio-economic variables researchers do not have much choice but to use them. Fourth, due to space limitations, the full examination of individual variables on the maps was not possible. All these limitations can be the object of other research papers.

On the whole, it is hoped that our quantitative analysis throws

${ }^{34}$ Even if some variables within each group (e.g. within the B group) are close to the variables of the other groups we regard this closeness as expected in a continuously changing and dynamic socio-economic environment.

${ }^{35}$ This is a general statement and it is possible that some variables within one group are highly correlated with some other variables in other groups. A multivariate statistical regression analysis might be possible in some cases (despite the smal number of countries and/or variables in our sample), but this could be the topic of another research paper. some evidence that any society's development is multi faced and multidimensional: distinct categories of socio-economic analysis all contribute in the final picture that we have about countries when we describe them only in terms of economic indexes of development such as GDP per capita. All these categories and their variables that constitute them move in tandem through time. We cannot consider the significance of economic factors of societal development by themselves without also considering institutional, governance, educational and in general social factors as well. However, we can describe a nation stage of overall societal development by considering simple indicators such GDP per capita or 'rule of law' or 'control of corruption', and so on. All this has important policy implications: first, the government' fundamental role in adjusting institutions in order to support business and economic performance. Secondly, business attitudes can be at least partially directed through adequate education towards higher socioeconomic performance. Third, positive cultural changes although slow to take place can be speeded up by the presence of all other positive changes in the society.

\section{Acknowledgments}

I am grateful to the research assistant Otgonbat Ishdagva for his valuable work on collecting and checking data as well as making graphs and Tables. Also earlie versions of this study received comments by scholars in conferences/seminars.

\section{References}

1. Baster N (1972) Development indicators: an introduction. The Journal of Development Studies 8: 1-20.

2. McGranahan D (1972) Development indicators and development models. The Journal of Development Studies 8: 91-102.

3. Adelman I, Morris CT (1965) A Factor Analysis of the Interrelationship between Social and Political Variables and Per Capita Gross National Product. The Quarterly Journal of Economics 79: 555-578.

4. Syrquin M (1978) The Application of Multidimensional Scaling to the Study of Economic Development. The Quarterly Journal of Economics 92: 621-639.

5. Cinca CS, Molinero CM, Larraz JLG (2002) A multivariate study of the economy of the European Union via financial statements analysis. Journal of the Royal Statistical Society: Series D (The Statistician) 51: 335-354.

6. Papalia RB, Bertarelli S (2010) Evaluating total factor productivity differences by a mapping structure in growth models. International Regional Science Review 33: 31-59.

7. Akkucuk U (2011) A Study on the Competitive Positions of Countries Using Cluster Analysis and Multidimensional Scaling. European Journal of Economics, Finance and Administrative Sciences 37: 17-26.

8. Chase RX (1985) A Theory of Socioeconomic Change: Entropic Processes Technology, and Evolutionary Development. Journal of Economic Issues 19 : 797-823.

9. Sanidas E (2006) The Open System of Four Dynamic Bio-socioeconomic Processes of the Firm: The Diamond of the Black Box. The Journal of Socioeconomics 35: 556-582.

10. Fedderke JW, Klitgaard R (1998) Economic growth and social indicators: an exploratory analysis. Economic Development and Cultural Change 46: 455-489.

11. Williamson OE (1998a) Transaction Cost Economics: How It Works; Where It is Headed. De Economist 146: 23-58.

12. North DC (1990) Institutions, Institutional Change, and Economic Performance, Cambridge University Press, Cambridge.

13. Granovetter M (1985) Economic Action and Social Structure: The Problem of Embeddedness. American Journal of Sociology 91: 481-510.

14. Hofstede G (2003) Culture's Consequences, Comparing Values, Behaviors, Institutions, and Organizations across Nations (2ndedn), Sage Publications, London.

15. Minkov M, Hofstede G (2011) The evolution of Hofstede's doctrine. Cross cultural management: An International Journal 18: 10-20. 
Citation: Sanidas E (2017) Evidence of the Strong Nexus between Economic, Social, Business, and Political Indicators across the World. Int J Econ Manag Sci 6: 481. doi: 10.4172/2162-6359.1000481

Page 24 of 24

16. Lian B, Oneal JR (1997) Cultural Diversity and Economic Development: A Cross-National Survey of 98 Countries, 1960-1985. Economic Development and Cultural Change 46: 61-77.

17. Mathers RL, Williamson CR (2011) Cultural Context: Explaining the Productivity of Capitalism, Kyklos 64: 231-252.

18. Williamson CR, Mathers RL (2011) Economic Freedom, culture, and growth, Public Choice 148: 313-335.

19. De Dios E (2008) Governance, Institutions and Political Economy: Abridged Version. Asian Development Bank (ADB) Draft Report, pp: 1-21.

20. Acemoglu D (2009) Introduction to Modern Economic Growth, Princeton and Oxford: Princeton University Press.

21. Niosi PJ (2008) Technology, development and innovation systems: An introduction. The Journal of Development Studies 44: 613-621.

22. Voigt $S$ (2009) The effects of competition policy on development-cross-country evidence using four new indicators. Journal of Development Studies 45: 1225 1248.

23. Landau D (2003) A simple theory of economic growth, economic development and cultural change 52: 217-235.

24. Knowles S, Garces-Ozanne A (2003) Government Intervention and Economic Performance in East Asia, Economic Development and Cultural Change 51: 451-477.

25. Norton SW (1992) Transaction Costs, Telecommunications, and the Microeconomics of Macroeconomic Growth. Economic Development and Cultural Change 41: 175-196.

26. Gallego I (2006) The use of economic, social, and environmental indicators as a measure of sustainable development in Spain. Corporate social responsibility and environmental management 13: 78-97.

27. Lewis $P$ (2004) Economics as Social Theory and the New Economic Sociology, in Lewis, Paul (ed) Transforming Economics: Perspectives on the Critical Realist Project, London \& New York: Routledge, pp. 167-186.

28. Polanyi K (1944) The Great Transformation, Rinehard \& Company, New York.

29. Jessop B (2001) Regulationist and Autopoieticist Reflections on Polanyi's Account of Market Economics and the Market Society. New Political Economy 6: 213-232.

30. Dixit A (2004) Lawlessness and economics: Alternative modes of Governance (Gorman Lectures in Economics, University College London), Princeton University Press, Princeton, NJ, USA

31. Kunneke RW (2008) Institutional Reform and Technological Practice: The case of electricity industrial and corporate changes 17: 233-265.

32. Key BD, Reed R, Sclar DA (2005) First-order Economizing: Organizational Adaptation and the Elimination of Waste in the US Pharmaceutical Industry. Journal of Managerial Issues 17: 511-527.

33. Boliari N (2007) Conceptualizing Institutions and Organizations: A Critical Approach. Journal of Business and Economic Research 5: 1-9.

34. Yeager T (1998) Institutions, Transition Economies, and Economic Development, Boulder, Colorado: Westview Press.

35. Seers D (1972) What are we trying to measure? The Journal of Development Studies 8: 21-36

36. Kruskal JB, Wish M (1978) Multidimensional Scaling, Beverly Hills and London: Sage Publications.

37. Hair JF, Black WC, Babin BJ, Anderson RE, Tatham RL (2006) Multivariate data analysis $\left(6^{\text {th }}\right.$ edtn), Pearson Prentice Hall, New Jersey, USA.

38. Craig SC, Douglas SP, Grein A (1992) Patterns of Convergence and Divergence among Industrialized Nations: 1960-1998. Journal of International Business Studies 23: 773-787.
39. Borg I, Groenen PJF (2010) Modern Multidimensional Scaling: Theory and Applications, Springer, New York, USA

40. Jessop RD (2006) State and Regulation-theoretical Perspectives on the European Union and the Failure of the Lisbon Agenda. Competition \& Change 10: 141-161.

41. Cyril E, Irma A (1967) Society, Politics and Economic Development, Baltimore: Johns Hopkins Press.

42. Cyril E, Irma A (2007) Analysis-of-Variance Techniques for the Study of Economic Development. Journal of Development Studies 8: 91-105.

43. Cyril E, Irma A (1974) The Derivation of Cardinal Scales from Ordinal Data: An Application of Mul-tidimensional Scaling to Measure Levels of National Development, in W. Sel-lekaerst, ed., Economic Development and Planning: Essays in Honour of Jan Tinbergen (London: Macmillan)

44. Agarwal M, Samanta S (2006) Structural Adjustment, Governance, Economic Growth and Social Progress. The Journal of International Trade \& Economic Development 15: 387-401.

45. Archibugi D, Coco A (2004) A New Indicator of Technological Capabilities for Developed and Developing Countries (ArCo). World Development 32: 629-654

46. Castaño M (2007) The influence of socioeconomic factors on economic growth International Advances in Economic Research 13: 139-145.

47. Chennareddy V (2010) Measures of income inequality, human brainpower capacity, social indicators, and their relationships in the United States. International Journal of Business and Public Administration 7: 131-147.

48. Corruption Perceptions Index of Heritage Foundation site (2017).

49. Fedderke JW, Luiz JM (2008) The political economy of institutions, stability and investment: a simultaneous equation approach in an emerging economy. The case of South Africa. The Journal of Development Studies 44: 1056-1079.

50. Global Competitiveness Report.

51. Hofstede' s Cultural Dimensions site: www.geert-hofstede.com/

52. Institute for Management Development (2008) IMD World Competitiveness Yearbook 2008, Lausanne, Switzerland: IMD International.

53. Knowles DS, Owen PD (2010) Which Institutions are good for Your Health? The Deep Determinants of Comparative Cross-country Health Status. The Journal of Development Studies 46: 701-723.

54. Langbein L, Knack S (2010) The Worldwide Governance Indicators: Six, One or None? The Journal of Development Studies 46: 350-370.

55. Lewis $P$ (2004) Transforming Economics: Perspectives on the Critical Realis Project, Routledge, London and New York.

56. Rayp G, Van DSN (2007) Measuring and explaining government efficiency in developing countries. Journal of Development Studies 43: 360-381.

57. Straub S (2011) Infrastructure and development: A critical Appraisal of the Macro-Level Literature. The Journal of Development Studies 47: 683-708.

58. Oliver EW (1998b) The Institutions of Governance. American Economic Review 88: 75-79.

59. Oliver EW (2003) Examining Economic Organization Through the Lens of Contract. Industrial and Corporate Change 12: 917-942.

60. Oliver EW (2005) The Economics of Governance. American Economic Review 95: 1-18.

61. Oliver EW (2008) Transaction Cost Economics: The Precursors. Economic Affairs $28: 8-14$

62. World Economic Forum (2017).

63. Worldwide Governance Indicators (2017)

64. World Values Surveys (2017). 